\title{
Endogenous biosynthesis of $n-3$ long-chain PUFA in Atlantic salmon
}

\author{
Thomas S. Mock ${ }^{1}$, David S. Francis ${ }^{1}$, Matthew K. Jago ${ }^{1}$, Brett D. Glencross ${ }^{2}$, Richard P. Smullen ${ }^{3}$ and \\ Giovanni M. Turchini ${ }^{1}$ \\ ${ }^{1}$ School of Life and Environmental Sciences, Deakin University, Geelong, VIC 3216, Australia \\ ${ }^{2}$ Institute of Aquaculture, University of Stirling, Stirling FK9 4LA, UK \\ ${ }^{3}$ Ridley Aqua-Feed Pty Ltd, Deception Bay, QLD 4504, Australia
}

(Submitted 14 October 2018 - Final revision received 25 January 2019 - Accepted 14 February 2019 - First published online 05 March 2019)

\begin{abstract}
A more efficient utilisation of marine-derived sources of dietary $n-3$ long-chain PUFA ( $n$-3 LC PUFA) in cultured Atlantic salmon (Salmo salar L.) could be achieved by nutritional strategies that maximise endogenous $n-3$ LC PUFA synthesis. The objective of the present study was to quantify the extent of $n$-3 LC PUFA biosynthesis and the resultant effect on fillet nutritional quality in large fish. Four diets were manufactured, providing altered levels of dietary $n-3$ substrate, namely, $18: 3 n-3$, and end products, namely, 20:5n-3 and $22: 6 n-3$. After $283 \mathrm{~d}$ of feeding, fish grew in excess of $3000 \mathrm{~g}$ and no differences in growth performance or biometrical parameters were recorded. An analysis of fatty acid composition and in vivo metabolism revealed that endogenous production of $n$ - 3 LC PUFA in fish fed a diet containing no added fish oil resulted in fillet levels of $n$-3 LC PUFA comparable with fish fed a diet with added fish oil. However, this result was not consistent among all treatments. Another major finding of this study was the presence of abundant dietary $n$ - 3 substrate, with the addition of dietary $n-3$ end product (i.e. fish oil) served to increase final fillet levels of $n$ - 3 LC PUFA. Specifically, preferential $\beta$-oxidation of dietary $\mathrm{C}_{18} n-3$ PUFA resulted in conservation of $n-3$ LC PUFA from catabolism. Ultimately, this study highlights the potential for endogenous synthesis of $n$-3 LC PUFA to, partially, support a substantial reduction in the amount of dietary fish oil in diets for Atlantic salmon reared in seawater.
\end{abstract}

Key words: Fatty acids: Metabolism: $\boldsymbol{n - 3}$ Fatty acids: Bioconversion: Aquaculture: Fish oil

The intense market volatility and reduced availability of traditional protein and lipid sources such as fishmeal and fish oil for use in aquafeed have led to constantly evolving dietary formulations ${ }^{(1-3)}$. The aquaculture industry has been active in adopting plant and animal protein and lipid sources for the incorporation into aquafeed; however, most of these ingredients contain none or considerably lower levels of the health beneficial fatty acids, namely, $n$-3 long-chain $(C \geq 20)$ PUFA (n-3 LC PUFA $)^{(2,4,5)}$. Consequently, maximising the deposition efficiency of increasingly limited dietary $n$ - 3 LC PUFA into the final edible product will prove integral to ensure the ongoing viability of the aquaculture sector ${ }^{(6-9)}$. The possible solutions available to address this challenge are based on the knowledge that changes to the fatty acid composition of the aquafeed influence not only the final fatty acid composition of fish fillets but also the various aspects of fatty acid metabolism, including in vivo fatty acid $\beta$-oxidation and bioconversion ${ }^{(10-13)}$. Concomitant with an increased understanding of fatty acid metabolism within popular cultured species in recent years, dietary formulations have been manipulated to promote the sparing of n-3 LC PUFA from catabolism as well as stimulate endogenous production via the $n-3$ LC PUFA biosynthetic pathway ${ }^{(7,9,10,14)}$. The $n$-3 LC PUFA biosynthetic pathway is facilitated by desaturase and elongase enzymes found in many fish and mammalian species ${ }^{(12,15,16)}$. However, the activity and efficiency of these enzymes in converting shorter $\left(C_{18}\right)$, less unsaturated fatty acids into longer $\left(\mathrm{C}_{20-22}\right)$, more unsaturated fatty acids are not uniform across species ${ }^{(12,17)}$. Specifically, carnivorous marine species of fish are purported to have a severely limited capacity to biosynthesise $22: 6 n-3$ from $18: 3 n-3$ dietary substrate ${ }^{(18-20)}$. It is hypothesised that the abundance of $n-3$ LC PUFA in marine ecosystems, which originate in lower trophic microbial and invertebrate organisms ${ }^{(21)}$, has rendered the $n$ - 3 LC PUFA biosynthetic pathway largely redundant ${ }^{(12,16,18,19)}$. Conversely, freshwater and anadromous fish have adapted to a relative paucity of dietary available $n-3$ LC PUFA and exhibit a much higher capacity to biosynthesise physiologically required $n-3$ LC PUFA from dietary $18: 3 n-3^{(22-25)}$.

Atlantic salmon (Salmo salar) have a demonstrated, albeit often limited, ability to bioconvert $18: 3 n-3$ to $22: 6 n-3^{(9,26,27)}$.

Abbreviation: $n-3$ LC PUFA, $n-3$ long-chain PUFA.

* Corresponding author: D. S. Francis, email d.francis@deakin.edu.au 
Consequently, numerous metabolic responses to dietary fatty acid changes have been elucidated in this spe$\operatorname{cies}^{(8,11,13,15,22,23,28-30)}$. It has been shown that endogenous production of $22: 6 n-3$ in Atlantic salmon is heavily influenced by the dietary ratio of shorter-chain to long-chain $n-3$ PUFA. Specifically, an increase in substrate (18:3n-3) availability appears to enhance the activity of the $\Delta-6$ desaturase enzyme necessary for the first and one of the last steps of the $n-3$ PUFA bioconversion pathway ${ }^{(10,31-34)}$. Conversely, the presence of end product (22:6n-3) may elicit a negative feedback mechanism on the pathway ${ }^{(33)}$. Furthermore, the extent of endogenous synthesis of $22: 6 n-3$ in Atlantic salmon may only occur to an extent that satisfies a minimum physiological requirement, which itself, is dictated by changing environmental conditions and life history stage ${ }^{(9,12,35)}$. For instance, $n-3$ LC PUFA synthesis is generally more efficient in the juvenile stages and then decreases in the seawater growth phase ${ }^{(36)}$. Therefore, endogenous synthesis has been documented to be unable to enrich the fillet tissue with $n-3$ LC PUFA to the same extent as dietary added fish oil in Atlantic salmon ${ }^{(13,24,37-41)}$. Nevertheless, there remains multiple, interrelated dietary and environmental factors that dictate the final concentration of $n-3$ LC PUFA that can be synthesised de novo ${ }^{(10,28,42-45)}$.

Currently, commercial aquafeed formulations for Atlantic salmon contain a physiological excess of $n-3$ LC PUFA to ensure a high level of deposition of these fatty acids into the fillet tissue. Atlantic salmon, in particular, are popular with consumers, in part, due to historically high levels of $n-3$ LC PUFA $^{(46-48)}$. However, this is becoming increasingly marginal due to mounting pressure from both within and outside the aquaculture industry for $n$-3 LC PUFA-rich oil sources. Levels of n-3 LC PUFA in farmed Atlantic salmon have followed a declining trend ${ }^{(4)}$ and are approaching the minimal levels needed to satisfy both, the physiological needs of the animal and consumer expectation. Therefore, novel approaches to maximise the deposition efficiency of nutritionally valuable fatty acids in this popular table fish are increasingly sought after $^{(2,7,49)}$. In the future, a more efficient utilisation of $n-3$ LC PUFA in cultured salmonids may be reliant on dietary strategies that maximise $n$-3 PUFA bioconversion ${ }^{(10,28,46)}$. Hence, further research that elucidates the potential to exploit fatty acid metabolism, namely, $n-3$ LC PUFA, bioconversion in large Atlantic salmon is warranted.

To date, the majority of laboratory-based fish trials have focused on smaller sub-market-sized fish due to logistical constraints. This limits commercial relevance as physiological fatty acid requirements, and by extension in vivo fatty acid metabolism, change in response to ontogenic development ${ }^{(46)}$. Furthermore, trials conducted on farm may compromise the accuracy of feed and fatty acid uptake measurements due to a typically higher food conversion ratio and difficulties quantifying uneaten feed ${ }^{(50)}$. Accordingly, the present experiment was conducted in a laboratory set-up with fish grown up to a marketable size. The trial utilised three oil sources, namely, fish oil, poultry by-product oil and camelina oil to create four experimental diets with varying levels of shorter-chain and long-chain $n-3$ PUFA. The objective, therefore, was to provide altered substrate (18:3n-3) and end product $(20: 5 n-3$ and $22: 6 n-3)$ ratios along the $n$ - 3 LC PUFA bioconversion pathway and subsequently quantify the extent of $n$ - 3 LC PUFA biosynthesis, towards enhancing current knowledge, and industry relevance, regarding the potential for endogenous production of $n-3$ LC PUFA in market-sized Atlantic salmon.

\section{Methods}

\section{Ethics statement}

All animals and procedures in this experiment were approved by the Deakin University Animal Welfare Committee (B252015). All possible steps to minimise animal suffering and provide an enriched environment were taken.

\section{Animals, trial facility and sampling}

Juvenile Atlantic salmon ( $S$. salar) were sourced from a commercial producer (Mountain Fresh Trout and Salmon Farm, Harrietville, VIC, Australia) and transported to the Deakin Aquaculture Futures Facility (Deakin University, Warrnambool Campus, VIC, Australia). Juvenile fish were acclimatised to the facility in freshwater before gradually being exposed to saltwater. Smoltification of fish was confirmed by plasma chloride analysis. Following smoltification, fish were acclimatised to the experimental conditions and maintained on a commercial $6 \mathrm{~mm}$ salmonid diet (Ridley Aquafeed Pty Ltd) before the commencement of the trial period. The experiment was conducted in a closed-loop, thermostatically controlled, recirculating aquaculture system containing eight (5000 litres) rearing tanks. Physical and biological filtration (drum filter fitted with a $60 \mu \mathrm{m}$ screen; Hydrotech) and UV disinfection maintained water quality throughout the experiment. The system was maintained on a $12 \mathrm{~h}$ light $-12 \mathrm{~h}$ dark cycle and temperature was kept at $15 \cdot 0 \pm 0 \cdot 5^{\circ} \mathrm{C}$ for the duration of the experiment. Dissolved oxygen was maintained at optimal conditions, and the levels of metabolic waste, total ammonia, $\mathrm{P}$, nitrite and nitrate were monitored daily using Aquamerck Test kits (Merck), which remained within acceptable limits throughout the trial. Immediately preceding the trial, an initial sample of six fish were euthanised in excess anaesthetic (AQUI-S, 0.05 ml/1) and stored at $-20^{\circ} \mathrm{C}$ until analysis. Initially, 272 fish were distributed amongst eight tanks (thirty-four fish per tank) and assigned one of four dietary treatments in duplicate (two tanks per treatment; $n$ 2, $N$ 8). After 98 and $196 \mathrm{~d}$, weight checks were performed and fish removed to improve stocking densities. Fish were fed to apparent satiation twice a day at 09.00 and $16.00 \mathrm{~h}$ for the entire $(283 \mathrm{~d})$ grow-out period. Feed consumption and mortalities $(<2 \%)$ were recorded throughout the trial and remained within acceptable limits. Faeces were collected 2 weeks before final sampling for digestibility estimation. At the completion of the grow-out phase, all fish were euthanised in excess anaesthetic (AQUI-S, 0.05 ml/l) and weighed. Subsequently, eighteen fish per treatment (nine fish per tank) were selected and stored at $-20^{\circ} \mathrm{C}$ until analysed. The sampled fish were separated into two groups: the first group (ten fish per treatment; five fish pooled per tank) was used for analysis of biometry and for chemical analysis of whole body, the second group (eight fish 
per treatment; four fish pooled per tank) was used for biometry and chemical analysis of fillet.

\section{Experimental diets}

Four experimental diets were formulated to be iso-lipidic $(310 \mathrm{mg} / \mathrm{g})$, iso-proteic $(440 \mathrm{mg} / \mathrm{g})$ and iso-energetic $(26 \mathrm{~kJ} / \mathrm{g})$ (Table 1). For manufacturing the four experimental diets, a $9 \mathrm{~mm}$ extruded pellet for Atlantic salmon was produced by a commercial feed producer (Ridley Aquafeed Pty Ltd) and removed from the production process at the feed mill postextrusion, before oil coating and delivered to Deakin University for subsequent vacuum oil coating of the experimental oils. A small-scale, laboratory-based, vacuum-coater equipped with a vacuum pump and rotation mechanism was used to add the dietary lipid. The four experimental diets were achieved using three different lipid sources, poultry by-product oil, fish oil and camelina oil, to create varied ratios of short-chain and longchain $n$-3 fatty acids and therefore four varied compositions of 'substrate' (18:3n-3) and 'end product' (20:5n-3 and $22: 6 n-3)$ in terms of in vivo bioconversion of $n$ - 3 fatty acids via the desaturation, elongation enzymatic pathway. The experimental oil blends were added to the 'uncoated' pellets and resulted in the following four diets:

- Low substrate:Low end product (Low:Low), where added oil consisted of $100 \%$ poultry by-product oil.

- Low substrate:High end product (Low:High), where added oil consisted of $80 \%$ poultry by-product oil and $20 \%$ fish oil.

- High substrate:Low end product (High:Low), where added oil consisted of $80 \%$ camelina oil and $20 \%$ poultry by-product oil

- High substrate:High end product (High:High), where added oil consisted of $80 \%$ camelina oil and $20 \%$ fish oil.

\section{Growth performance, chemical analyses and fatty} acid analysis

Standard formulae were used to assess growth, feed utilisation and biometrical data. These included initial and final average weight, weight gain $(\mathrm{g})$, weight gain $\%$, feed conversion ratio (FCR), specific growth rate (SGR), Fulton's condition factor $(K)$, dress-out percentage, fillet yield percentage (FY\%), hepatosomatic index (HSI\%) and viscera-somatic index (VSI \%). Calculations for these common formulae are presented in detail in Francis et al. ${ }^{(51)}$. The chemical composition of the experimental diets, faeces and fish samples was determined via proximate composition analysis according to standard methods, as previously described by Emery et al. ${ }^{(52)}$. Briefly, moisture was determined by drying samples in an oven at $80^{\circ} \mathrm{C}$ to a constant weight, while ash was determined by incinerating samples in a muffle furnace (S.E.M. (S.A.) Pty Ltd) at $550^{\circ} \mathrm{C}$ for $18 \mathrm{~h}$. Protein (Kjeldahl N: N $\left.\times 6.25\right)$ content was determined using an automated Kjeltech 2300 (Foss Tecator) and lipid was determined by solvent extraction ${ }^{(53)}$, where dichloromethane was used to replace chloroform for safety considerations. Following lipid extraction, fatty acids were esterified into methyl esters using an acid-catalysed methylation method and then analysed by GC as described in Norambuena et al. ${ }^{(54)}$. Resulting peaks were identified relative to known external standards and then corrected for theoretical flame ionisation detector response and methyl transformation required for GC analysis. The resulting quantitative fatty acid data were eventually reported as $\mathrm{mg} / \mathrm{g}$ for the experimental diets and as $\mu \mathrm{mol} / \mathrm{g}$ tissue (wet weight) for fillet tissue, as recommended by Parrish ${ }^{(55)}$

\section{Apparent nutrient digestibility and metabolism calculations}

Apparent digestibility was determined following methods presented in Atkinson et al. ${ }^{(56)}$, using ash instead of acid-insoluble ash as the inert marker. The calculation of apparent in vivo fatty acid metabolism was performed using the whole-body fatty acid balance method, as initially proposed and described by Turchini et al. ${ }^{(57)}$ with further development ${ }^{(34,58)}$. The results of the whole-body fatty acid balance method are reported as $\mathrm{nmol} / \mathrm{g}$ per $\mathrm{d}$ and percentage of net intake as presented in Norambuena et al. ${ }^{(11)}$.

\section{Statistical analysis}

All data were reported as means with their standard errors ( $n 2$, $N$ 8). After confirmation of normality and homogeneity of variance, data were subjected to two-way ANOVA to assess the effects of dietary substrate, dietary end product and dietary substrate by end product interactions. A Tukey's post hoc test determined statistical significance between homogenous subsets and was performed where statistical significant differences were identified. The analysis was performed using IBM SPSS Statistics version 24.0 (SPSS Inc.). Significance was accepted at $P<0.05$, where * $P<0.05 ;{ }^{* *} P<0.01$ and **** $P<0.001$.

Before the experiment, based on recent research with a similar study design and similar sized fish ${ }^{(59)}$, a between-group, within-treatment standard deviation of 197.4 was expected for an outcome of importance, specifically, final fish weight. Power analysis revealed that the minimum detectable difference in final weight between treatment groups given the sample size in the present study and assuming a statistical power of $80 \%$ was $262 \mathrm{~g}$. This was considered acceptable for the present study, considering the expectedly large final weights of the fish. Power analysis was performed using GraphPad StatMate version 2.0 (Graphpad Software Inc.).

\section{Results}

Diets

The four experimental diets were iso-energetic and proximate compositions were similar (Table 1 ). Total fatty acid concentration ranged from 247.4 to $272.6 \mathrm{mg} / \mathrm{g}$ diet in High:Low and Low:Low, respectively. Levels of individual fatty acids varied according to lipid source. Accordingly, Low:Low and Low:High were characterised by higher levels of both SFA and MUFA due to typically high $18: 1 n-9$ concentrations in poultry by-product oil. Total PUFA levels were higher in High:Low and High:High; however, $n$-6 PUFA was relatively consistent 
between dietary treatments. Levels of $n$-3 PUFA were markedly higher in High:Low and High:High, owing to high levels of 18:3n-3. Diet $n$-3 LC PUFA levels were predictably dictated by fish oil inclusion and were higher in Low:High and High:High owing to higher $22: 6 n-3$ concentrations. The ratio of $n-6: n-3$ fatty acids varied considerably between treatments, ranging from 0.8 to 4.1 in High:High and Low:Low, respectively $(P<0.05)$.

\section{Growth, feed utilisation parameters and biometric data}

Mortality rates were low during the trial and unrelated to diet. Diets were generally well accepted by fish. However, some maturation of fish occurred in the latter stages of the trial, which led to poorer than expected growth and food conversion. Fish gained approximately $2000 \mathrm{~g}$ and grew to weights in excess of $3000 \mathrm{~g}$, with no statistical differences evident between treatments. FCR ranged from 1.14 to 1.26 in Low:Low and High:High, respectively (Table 2) $(P>0.05)$. Overall, there were no significant differences in either growth or biometry measures between dietary treatments, including SGR, FCR, feed ration \% (relative to body mass), $K, \mathrm{FY} \%$ HSI $\%$ and VSI \%. There were no significant effects of dietary $n-3$ substrate, end product or substrate by end product interactions recorded with regard to any growth performance parameters.

Table 1. Proximate composition, total fatty acids (FA) and FA (mg/g diet) composition of the four experimental diets

\begin{tabular}{|c|c|c|c|c|}
\hline & \multicolumn{4}{|c|}{ Diets $^{*}$} \\
\hline & Low:Low & Low:High & High:Low & High:High \\
\hline \multicolumn{5}{|c|}{ Proximate composition $(\mathrm{mg} / \mathrm{g})$} \\
\hline Moisture & 39.8 & $39 \cdot 0$ & 38.6 & $45 \cdot 7$ \\
\hline Protein & $430 \cdot 6$ & $432 \cdot 8$ & $440 \cdot 7$ & $446 \cdot 4$ \\
\hline Lipid & $320 \cdot 2$ & 328.3 & 311.8 & 322.4 \\
\hline NFE & $176 \cdot 4$ & $165 \cdot 6$ & $173 \cdot 4$ & $155 \cdot 8$ \\
\hline Ash & 71.8 & $72 \cdot 5$ & $72 \cdot 8$ & $74 \cdot 3$ \\
\hline Energy $(\mathrm{kJ} / \mathrm{g})$ & $25 \cdot 8$ & $26 \cdot 0$ & $25 \cdot 7$ & $26 \cdot 0$ \\
\hline Total FA (mg/g diet) $\dagger$ & $272 \cdot 6$ & $270 \cdot 6$ & 247.4 & 253.7 \\
\hline SFA & 83.2 & $86 \cdot 4$ & $55 \cdot 8$ & $61 \cdot 8$ \\
\hline $14: 0$ & $3 \cdot 3$ & $5 \cdot 8$ & $2 \cdot 0$ & 4.4 \\
\hline $16: 0$ & $59 \cdot 8$ & $59 \cdot 6$ & $38 \cdot 3$ & $40 \cdot 1$ \\
\hline $18: 0$ & $17 \cdot 7$ & 17.5 & $12 \cdot 2$ & $12 \cdot 7$ \\
\hline Other SFA & 2.4 & 3.6 & 3.3 & 4.6 \\
\hline MUFA & 143.0 & $132 \cdot 7$ & $115 \cdot 3$ & $108 \cdot 1$ \\
\hline $16: 1 n-7$ & $14 \cdot 1$ & 14.7 & $8 \cdot 2$ & $9 \cdot 0$ \\
\hline $18: 1 n-9$ & 118.9 & $105 \cdot 6$ & $82 \cdot 7$ & 71.5 \\
\hline $18: 1 n-7$ & $6 \cdot 6$ & 6.9 & 4.6 & $5 \cdot 0$ \\
\hline $20: 1 n-9$ & $1 \cdot 7$ & $2 \cdot 4$ & $14 \cdot 8$ & $16 \cdot 2$ \\
\hline Other MUFA§ & 1.7 & 3.0 & 4.9 & $6 \cdot 3$ \\
\hline Total trans-FAll & 1.7 & 1.5 & 0.8 & 0.6 \\
\hline PUFA & 44.7 & 49.9 & 75.5 & 83.2 \\
\hline $18: 2 n-6$ & $34 \cdot 1$ & 29.7 & $36 \cdot 3$ & 33.2 \\
\hline $20: 2 n-6$ & 0.3 & 0.3 & 1.8 & 1.9 \\
\hline $20: 4 n-6$ & 0.6 & 1.3 & 0.4 & 1.0 \\
\hline Other $n-6$ PUFAT & 0.8 & $1 \cdot 2$ & 0.6 & $1 \cdot 1$ \\
\hline$n-6$ PUFA & $35 \cdot 8$ & $32 \cdot 6$ & 39.2 & $37 \cdot 3$ \\
\hline $18: 3 n-3$ & $5 \cdot 3$ & 4.7 & $32 \cdot 3$ & 33.5 \\
\hline $20: 5 n-3$ & 1.0 & 4.6 & 0.8 & 4.2 \\
\hline $22: 5 n-3$ & 0.4 & 1.0 & 0.3 & 0.9 \\
\hline $22: 6 n-3$ & 1.5 & 5.5 & $1 \cdot 2$ & 4.9 \\
\hline$n-3$ PUFA & $8 \cdot 7$ & $16 \cdot 6$ & $36 \cdot 2$ & $45 \cdot 2$ \\
\hline Other $n-3$ PUFA $^{\star \star}$ & 0.5 & 0.8 & 1.6 & $1 \cdot 7$ \\
\hline LC PUFA & $4 \cdot 6$ & $14 \cdot 2$ & 6.5 & $15 \cdot 5$ \\
\hline$n-6$ LC PUFA & 1.4 & 2.5 & $2 \cdot 7$ & 3.9 \\
\hline$n-3$ LC PUFA & $3 \cdot 2$ & 11.7 & $3 \cdot 8$ & 11.6 \\
\hline$n-6: n-3$ ratio $\dagger$ & $4 \cdot 1$ & $2 \cdot 0$ & $1 \cdot 1$ & 0.8 \\
\hline
\end{tabular}

NFE, N-free extract; LC, long chain.

* Diets: Low:Low = low substrate, low end product diet consisting of $43 \%$ protein and $32 \%$ lipid, added oil consists of $100 \%$ poultry by-product oil; Low:High = low substrate, high end product diet consisting of $43 \%$ protein and $33 \%$ lipid, added oil consists of $80 \%$ poultry by-product oil and $20 \%$ fish oil; High:Low = high substrate, low end product diet consisting of $44 \%$ protein and $31 \%$ lipid, added oil consists of $80 \%$ camelina oil and $20 \%$ poultry by-product oil; High:High = high substrate, high end product diet consisting of $45 \%$ protein and $32 \%$ lipid, added oil consists of $80 \%$ camelina oil and $20 \%$ fish oil.

$\dagger$ Total FA $=$ total FA $\mathrm{mg} / \mathrm{g}$ of diet.

$\ddagger$ Other SFA $=$ sum of $12: 0,15: 0,17: 0,20: 0,22: 0$ and $24: 0$.

$\S$ Other MUFA $=$ sum of $14: 1 n-5,15: 1 n-5,17: 1 n-7,20: 1 n-13,20: 1 n-11,22: 1 n-11,22: 1 n-9$ and $24: 1 n-9$.

II Total trans-FA $=$ sum of $18: 1 n-9 t, 18: 1 n-7 t$ and $18: 2 n-6 t$.

T Other $n-6$ PUFA $=$ sum of $18: 3 n-6,20: 2 n-6,20: 3 n-6,22: 2 n-6,22: 4 n-6$ and $22: 5 n-6$.

** Other $n-3$ PUFA $=$ sum of $20: 3 n-3,20: 4 n-3,24: 5 n-3$ and $24: 6 n-3$.

†† $n-6: n-3$ ratio $=$ ratio between $n-6$ PUFA and $n-3$ PUFA 


\section{Apparent nutrient and fatty acid digestibility}

High nutrient digestibility values (apparent digestibility coefficient \%) were observed across treatments, with only one significant difference recorded, where N-free extract digestibility was higher in Low:Low in comparison with High:High $(P<0.05)$ (Table 3$)$. Fatty acid digestibility values were high with no significant differences recorded between treatments, though there was a significant effect of end product recorded for the digestibility of $20: 5 n-3$.

\section{Tissue proximate and fillet fatty acid composition}

No significant differences in fillet proximate composition were evident (Table 4), even if fillet lipid concentrations varied between treatments, ranging from 75.1 to $104.7 \mathrm{mg} / \mathrm{g}$ tissue in Low:High and High:High, respectively $(P>0.05)$. Owing to differences in fillet lipid, total fatty acid concentration was lowest in Low:High and highest in High:High, though differences were not significant $(P>0 \cdot 05)$. Expectedly, fillet levels of 22: 6n-3 were higher in High:High compared with Low:Low in terms of both $\mu \mathrm{mol} / \mathrm{g}$ of fillet tissue and $\mathrm{mg} / 100 \mathrm{~g}$ of fillet $(P<0.05)$. However, High:Low had comparable levels of 22 : 6n-3 compared with both Low:High and High:High $(P>0.05)$. Furthermore, High:Low had marginally higher levels of total $n-3$ LC PUFA compared with Low:High, despite the latter treatment diet containing no added fish oil $(P>0 \cdot 05)$. As expected, High: High had higher levels of $n$-3 LC PUFA compared with Low:Low $(P<0 \cdot 05)$. The $n-6: n-3$ PUFA ratios varied across treatments and followed dietary trends, ranging from 1.0 to 2.7 in High:High and Low:Low, respectively $(P<0 \cdot 05)$. Across treatments, there was a clear effect of substrate and end product recorded for $20: 5 n-3$, $22: 5 n-3$ and total $n-3$ LC PUFA in terms of both $\mu \mathrm{mol} / \mathrm{g}$ of fillet tissue and $\mathrm{mg} / 100 \mathrm{~g}$ of fillet; however, no significant substrate by end product interaction was recorded.

\section{Apparent in vivo fatty acid metabolism}

Apparent in vivo fatty acid $\beta$-oxidation (expressed as nmol of fatty acid $\beta$-oxidised, per gram of fish per $\mathrm{d} ; \mathrm{nmol} / \mathrm{g}$ per $\mathrm{d}$ and as percentage of net intake) (Table 6 and Fig. 1, respectively) as calculated by the whole-body fatty acid balance method was highest in 16:0 and 18:1n-9 across all treatments, relative to high dietary inclusion levels. Accordingly, there was a significant effect of substrate and end product concentration on the $\beta$-oxidation of SFA $(P<0.001$ and $P<0 \cdot 01$, respectively). MUFA was $\beta$-oxidised to a similar extent as SFA where treatments demonstrated a similar trend; however, no significant differences were recorded between treatments $(P>0 \cdot 05)$. Substrate concentration had a clear effect of the $\beta$-oxidation of 18:3n-3 $(P<0 \cdot 001)$, where the High:Low and High:High treatments recorded significantly higher $\beta$-oxidation of $18: 3 n-3$ $(P<0.05)$. However, in terms of $\beta$-oxidation calculated based the percentage of net intake, both the low substrate treatments (Low:Low and Low:High) recorded higher values (approximately $70 \%$ ). Notably, $22: 6 n-3$ was highly conserved across treatments and recorded low $\beta$-oxidation values. 
Table 3. Nutrient and fatty acids (FA) digestibility (apparent digestibility coefficient, \%) of the four experimental diets in Atlantic salmon* (Mean values with their standard errors, $n 2, N 8$ )

\begin{tabular}{|c|c|c|c|c|c|c|c|c|c|c|c|}
\hline & \multicolumn{8}{|c|}{ Diets† } & & & \\
\hline & \multicolumn{2}{|c|}{ Low:Low } & \multicolumn{2}{|c|}{ Low:High } & \multicolumn{2}{|c|}{ High:Low } & \multicolumn{2}{|c|}{ High:High } & \multicolumn{3}{|c|}{ Effect of substrate, end product and substrate/end product interaction $(P)$} \\
\hline & Mean & SEM & Mean & SEM & Mean & SEM & Mean & SEM & Substrate & End product & Interaction \\
\hline \multicolumn{12}{|l|}{ Nutrients } \\
\hline DM & $86 \cdot 2$ & 0.2 & 84.6 & 0.3 & $85 \cdot 0$ & 0.2 & 83.9 & $1 \cdot 1$ & NS & NS & NS \\
\hline Protein & 94.8 & 0.0 & 93.3 & 0.4 & 93.5 & 0.3 & 93.3 & 0.8 & NS & NS & NS \\
\hline Lipid & 79.9 & 3.2 & $82 \cdot 3$ & $2 \cdot 3$ & 84.7 & 0.3 & 81.7 & 3.4 & NS & NS & NS \\
\hline NFE & $81.9^{\mathrm{a}}$ & 0.1 & $76 \cdot 6^{a, b}$ & 0.1 & $78 \cdot 4^{\mathrm{a}, \mathrm{b}}$ & 1.6 & $73 \cdot 7^{\mathrm{b}}$ & $2 \cdot 1$ & NS & NS & NS \\
\hline Energył & 94.1 & 0.3 & $92 \cdot 8$ & 0.4 & 93.3 & 0.2 & 92.2 & 1.2 & NS & NS & NS \\
\hline Total FA & $96 \cdot 8$ & 0.6 & 96.5 & 0.1 & $97 \cdot 2$ & 0.4 & $96 \cdot 1$ & 0.9 & NS & NS & NS \\
\hline \multicolumn{12}{|l|}{ FA } \\
\hline $14: 0$ & 96.5 & 0.3 & $95 \cdot 7$ & 0.1 & 96.0 & 0.3 & $95 \cdot 3$ & 0.5 & NS & NS & NS \\
\hline $16: 0$ & 94.7 & 0.2 & 93.8 & 0.2 & 94.6 & 0.3 & 94.2 & 0.3 & NS & NS & NS \\
\hline $18: 0$ & $92 \cdot 8$ & 0.1 & 91.8 & 0.3 & $92 \cdot 4$ & 0.3 & 92.6 & 0.1 & NS & NS & $<0.05$ \\
\hline $16: 1 n-7$ & 98.8 & 0.5 & 98.7 & 0.2 & 98.9 & 0.2 & 98.0 & 0.8 & NS & NS & NS \\
\hline $18: 1 n-9$ & 97.5 & 0.9 & 97.6 & 0.1 & 97.9 & 0.4 & $96 \cdot 3$ & 1.4 & NS & NS & NS \\
\hline $18: 1 n-7$ & 97.3 & 0.9 & $97 \cdot 3$ & 0.1 & 97.5 & 0.4 & 95.8 & 1.5 & NS & NS & NS \\
\hline $20: 1 n-9$ & 95.9 & 0.8 & $96 \cdot 0$ & 0.1 & $96 \cdot 6$ & 0.6 & 94.4 & 1.9 & NS & NS & NS \\
\hline $18: 2 n-6$ & 98.5 & 0.7 & 98.7 & 0.1 & 98.7 & 0.3 & 97.7 & 0.8 & NS & NS & NS \\
\hline $20: 2 n-6$ & 95.8 & 0.9 & 95.4 & 0.5 & 97.4 & 0.5 & 95.5 & 1.6 & NS & NS & NS \\
\hline $20: 4 n-6$ & $97 \cdot 8$ & 0.7 & 98.8 & 0.2 & 96.5 & $1 \cdot 3$ & $98 \cdot 3$ & 0.1 & NS & NS & NS \\
\hline $18: 3 n-3$ & 98.8 & 0.6 & 98.9 & 0.1 & 98.9 & 0.2 & 98.0 & 0.7 & NS & NS & NS \\
\hline $20: 5 n-3$ & 98.8 & 0.3 & 99.5 & 0.0 & 98.2 & 0.4 & 99.1 & 0.1 & NS & $<0.05$ & NS \\
\hline $22: 5 n-3$ & 96.9 & 0.0 & 98.6 & 0.0 & $96 \cdot 2$ & $1 \cdot 1$ & $97 \cdot 7$ & 0.3 & NS & NS & NS \\
\hline $22: 6 n-3$ & 97.0 & 1.0 & 98.7 & 0.2 & 95.0 & 1.8 & $97 \cdot 7$ & 0.1 & NS & NS & NS \\
\hline
\end{tabular}

NFE, N-free extract.

a,b Mean values within a row with unlike superscript letters were significantly different $(P<0.05)$

*Treatment means were analysed by one-way ANOVA with Tukey's post hoc test of multiple comparisons. Effects of substrate, end product and substrate/end product interaction were analysed by two-way ANOVA. † See Table 1 for experimental diet abbreviations.

‡ Calculated on the basis of $23 \cdot 6,39.5$ and $17.2 \mathrm{~kJ} / \mathrm{g}$ of protein, fat and carbohydrate, respectively.

$\S$ Value of $100=F A$ not detected in faeces. 
Table 4. Proximate ( $\mathrm{mg} / \mathrm{g}$ of tissue) and fatty acid $(\mathrm{FA})$ composition ( $\mu \mathrm{mol} / \mathrm{g}$ tissue) of fillets of Atlantic salmon fed the four experimental diets for $283 \mathrm{~d}^{*}$ (Mean values with their standard errors, $n 2, N 8$ )

\begin{tabular}{|c|c|c|c|c|c|c|c|c|c|c|c|}
\hline & \multicolumn{8}{|c|}{ Diets† } & & & \\
\hline & \multicolumn{2}{|c|}{ Low:Low } & \multicolumn{2}{|c|}{ Low:High } & \multicolumn{2}{|c|}{ High:Low } & \multicolumn{2}{|c|}{ High:High } & \multicolumn{3}{|c|}{ Effect of substrate, end product and substrate/end product interaction $(P)$} \\
\hline & Mean & SEM & Mean & SEM & Mean & SEM & Mean & SEM & Substrate & End product & Interaction \\
\hline \multicolumn{12}{|c|}{ Proximate composition (mg/g of tissue) } \\
\hline Moisture & $692 \cdot 4$ & 13.9 & 699.3 & $5 \cdot 8$ & 683.8 & $18 \cdot 2$ & 677.9 & 6.6 & NS & NS & NS \\
\hline Protein & 207.9 & 1.9 & 214.0 & $2 \cdot 3$ & 206.5 & 1.3 & 205.5 & $5 \cdot 2$ & NS & NS & NS \\
\hline Lipid & $92 \cdot 1$ & $17 \cdot 3$ & $75 \cdot 1$ & 3.7 & 97.9 & $16 \cdot 6$ & 104.7 & 3.1 & NS & NS & NS \\
\hline Ash & $8 \cdot 1$ & 0.5 & $8 \cdot 7$ & 0.1 & $9 \cdot 4$ & 0.1 & 8.7 & $1 \cdot 1$ & NS & NS & NS \\
\hline \multicolumn{12}{|l|}{$\mathrm{FA}(\mu \mathrm{mol} / \mathrm{g}$ of tissue) } \\
\hline Total FA ( $\mu \mathrm{g} / \mathrm{g}$ of tissue) & $277 \cdot 1$ & $52 \cdot 4$ & 221.9 & $10 \cdot 0$ & 296.6 & $54 \cdot 7$ & 321.4 & 11.5 & NS & NS & NS \\
\hline SFA & 61.5 & $10 \cdot 8$ & $50 \cdot 2$ & 0.6 & $59 \cdot 6$ & $10 \cdot 9$ & $66 \cdot 1$ & 3.0 & NS & NS & NS \\
\hline $14: 0$ & 3.8 & 0.7 & 4.0 & 0.0 & 3.4 & 0.7 & 5.4 & 0.3 & NS & NS & NS \\
\hline $16: 0$ & $43 \cdot 1$ & 7.5 & $34 \cdot 3$ & 0.2 & 41.0 & 7.8 & $44 \cdot 7$ & $2 \cdot 4$ & NS & NS & NS \\
\hline $18: 0$ & $13 \cdot 1$ & $2 \cdot 2$ & $10 \cdot 2$ & 0.4 & $12 \cdot 8$ & $2 \cdot 0$ & $13 \cdot 2$ & 0.2 & NS & NS & NS \\
\hline Other SFA§ & 1.6 & 0.3 & 1.6 & 0.0 & $2 \cdot 3$ & 0.4 & $2 \cdot 8$ & 0.1 & $<0.05$ & NS & NS \\
\hline MUFA & 161.5 & $32 \cdot 3$ & 120.9 & $6 \cdot 7$ & 153.4 & 28.0 & 155 & 4.1 & NS & NS & NS \\
\hline $16: 1 n-7$ & 14.5 & 3.3 & 11.9 & 0.2 & 11.6 & $2 \cdot 3$ & 13.4 & 0.7 & NS & NS & NS \\
\hline $18: 1 n-9$ & 129.2 & $25 \cdot 8$ & 94.2 & 5.5 & $111 \cdot 1$ & $20 \cdot 0$ & 107.9 & $2 \cdot 3$ & NS & NS & NS \\
\hline $18: 1 n-7$ & 8.7 & 1.7 & $7 \cdot 3$ & 0.5 & $7 \cdot 1$ & 1.3 & 7.9 & 0.2 & NS & NS & NS \\
\hline $20: 1 n-9$ & $6 \cdot 0^{\mathrm{a}}$ & 0.9 & $5 \cdot 0^{\mathrm{a}}$ & 0.5 & $13.9^{\mathrm{b}}$ & $2 \cdot 2$ & $17 \cdot 0^{\mathrm{b}}$ & 0.1 & $<0.01$ & NS & NS \\
\hline Other MUFAll & $3 \cdot 2^{a, b}$ & 0.6 & $2 \cdot 4^{\mathrm{a}}$ & 0.0 & $9.7^{\mathrm{b}}$ & $2 \cdot 2$ & $8 \cdot 8^{a, b}$ & 1.0 & $<0.01$ & NS & NS \\
\hline Total trans-FA & 1.2 & 0.2 & 0.8 & 0.0 & 0.9 & 0.2 & 0.9 & 0.0 & NS & NS & NS \\
\hline PUFA & $52 \cdot 8$ & $9 \cdot 1$ & 49.9 & $2 \cdot 7$ & 82.6 & $15 \cdot 6$ & $99 \cdot 3$ & 4.3 & $<0.05$ & NS & NS \\
\hline $18: 2 n-6$ & $27 \cdot 7$ & $5 \cdot 0$ & $23 \cdot 3$ & $1 \cdot 3$ & $36 \cdot 7$ & $7 \cdot 8$ & 39.2 & 1.7 & NS & NS & NS \\
\hline $20: 2 n-6$ & $1.9^{\mathrm{a}, \mathrm{b}}$ & 0.2 & $1 \cdot 7^{\mathrm{a}}$ & 0.2 & $2.9^{\mathrm{a}, \mathrm{b}}$ & 0.6 & $3 \cdot 6^{\mathrm{b}}$ & 0.0 & $<0.05$ & NS & NS \\
\hline $20: 4 n-6$ & 2.8 & 0.5 & $2 \cdot 0$ & 0.1 & 1.9 & 0.2 & $2 \cdot 0$ & 0.1 & NS & NS & NS \\
\hline Other $n-6$ PUFAף & $6 \cdot 1$ & 1.3 & 3.4 & 0.3 & $5 \cdot 7$ & 0.8 & 4.4 & 0.0 & NS & NS & NS \\
\hline$n-6$ PUFA & 38.5 & $7 \cdot 0$ & $30 \cdot 3$ & 1.8 & $47 \cdot 1$ & $9 \cdot 4$ & $49 \cdot 2$ & 1.8 & NS & NS & NS \\
\hline$n-6$ LC PUFA & 8.8 & 1.5 & $6 \cdot 3$ & 0.5 & 8.0 & 1.0 & 8.6 & 0.1 & NS & NS & NS \\
\hline $18: 3 n-3$ & $2 \cdot 2^{\mathrm{a}}$ & 0.4 & $2 \cdot 3^{\mathrm{a}}$ & 0.1 & $17 \cdot 3^{\mathrm{b}}$ & 4.4 & $24 \cdot 2^{b}$ & 2.2 & $<0.01$ & NS & NS \\
\hline $20: 5 n-3$ & $1.5^{\mathrm{a}}$ & 0.3 & $2 \cdot 2^{\mathrm{a}}$ & 0.0 & $3 \cdot 1^{a, b}$ & 0.5 & $4 \cdot 3^{\mathrm{b}}$ & 0.3 & $<0.01$ & $<0.05$ & NS \\
\hline $22: 5 n-3$ & $0.8^{\mathrm{a}}$ & 0.1 & $1 \cdot 3^{\mathrm{a}, \mathrm{b}}$ & 0.1 & $1.5^{b}$ & 0.0 & $2 \cdot 1^{\mathrm{c}}$ & 0.0 & $<0.01$ & $<0.01$ & NS \\
\hline $22: 6 n-3$ & $9.0^{\mathrm{a}}$ & 1.0 & $12 \cdot 3^{a, b}$ & 0.7 & $10 \cdot 4^{\mathrm{a}, \mathrm{b}}$ & 0.7 & $14 \cdot 1^{\mathrm{b}}$ & 0.1 & NS & $<0.01$ & NS \\
\hline Other $n$-3 PUFA†† & $0.7^{\mathrm{a}}$ & 0.1 & $0.9^{a}$ & 0.0 & $3.0^{\mathrm{b}}$ & 0.5 & $4.7^{\mathrm{b}}$ & 0.1 & $<0.001$ & $<0.05$ & $<0.05$ \\
\hline$n-3$ PUFA & $14 \cdot 1^{\mathrm{a}}$ & 1.9 & $19 \cdot 0^{a, b}$ & 0.9 & $35 \cdot 2^{\mathrm{b}, \mathrm{c}}$ & $6 \cdot 1$ & $49 \cdot 4^{\mathrm{C}}$ & $2 \cdot 4$ & $<0.01$ & NS & NS \\
\hline$n-3$ LC PUFA & $11.8^{\mathrm{a}}$ & 1.5 & $16 \cdot 7^{\mathrm{a}}$ & 0.8 & $17 \cdot 9^{a}$ & 1.6 & $25 \cdot 1^{\mathrm{b}}$ & 0.2 & $<0.01$ & $<0.01$ & NS \\
\hline LC PUFA & $20 \cdot 6^{\mathrm{a}}$ & 3.0 & $23 \cdot 0^{a, b}$ & 1.4 & $25 \cdot 9^{a, b}$ & $2 \cdot 7$ & $33.7^{\mathrm{b}}$ & 0.3 & $<0.05$ & NS & NS \\
\hline
\end{tabular}

LC, long chain.

a,b,c Mean values within a row with unlike superscript letters were significantly different $(P<0.05)$.

* Treatment means were analysed by one-way ANOVA with Tukey's post hoc test of multiple comparisons. Effects of substrate, end product and substrate/end product interaction were analysed by two-way ANOVA.

† See Table 1 for experimental diet abbreviations.

$\S$ Other SFA = sum of $12: 0,15: 0,17: 0,20: 0,21: 0,22: 0$ and $24: 0$.

II Other MUFA = sum of $14: 1 n-5,15: 1 n-5,17: 1 n-7,20: 1 n-11,22: 1 n-11$ and $24: 1 n-9$

T) Other $n-6$ PUFA $=$ sum of $18: 3 n-6,20: 3 n-6,22: 2 n-6,22: 4 n-6,22: 5 n-6$.

t† Other $n-3$ PUFA $=$ sum of $18: 4 n-3,20: 4 n-3,22: 3 n-3,24: 5 n 3$ and $24: 6 n-3$ 
Table 5. Fillet fatty acid composition (as $\mathrm{mg} / 100 \mathrm{~g}$ of edible product) of Atlantic salmon fillet fed the four experimental diets for $283 \mathrm{~d}^{*}$ (Mean values with their standard errors, $n 2, N 8$ )

\begin{tabular}{|c|c|c|c|c|c|c|c|c|c|c|c|}
\hline \multirow[b]{3}{*}{$\mathrm{mg} / 100 \mathrm{~g}$ of fillet } & \multicolumn{8}{|c|}{ Diets† } & & & \\
\hline & \multicolumn{2}{|c|}{ Low:Low } & \multicolumn{2}{|c|}{ Low:High } & \multicolumn{2}{|c|}{ High:Low } & \multicolumn{2}{|c|}{ High:High } & \multicolumn{3}{|c|}{ Effect of substrate, end product and substrate/end product interaction $(P)$} \\
\hline & Mean & SEM & Mean & SEM & Mean & SEM & Mean & SEM & Substrate & End product & Interaction \\
\hline $20: 5 n-3$ & $44 \cdot 2^{\mathrm{a}}$ & 7.9 & $67 \cdot 1^{\mathrm{a}}$ & $1 \cdot 1$ & $93 \cdot 0^{\mathrm{a}, \mathrm{b}}$ & 13.8 & $130 \cdot 5^{\mathrm{b}}$ & $7 \cdot 8$ & $<0.01$ & $<0.05$ & NS \\
\hline $22: 5 n-3$ & $27 \cdot 2^{\mathrm{a}}$ & 4.6 & $42 \cdot 8^{\mathrm{a}}$ & 2.8 & $48 \cdot 7^{\mathrm{a}}$ & 0.7 & $69 \cdot 8^{\mathrm{b}}$ & 0.6 & $<0.01$ & $<0.01$ & NS \\
\hline $22: 6 n-3$ & $294.5^{a}$ & 31.7 & $404 \cdot 9^{\mathrm{a}, \mathrm{b}}$ & 24.5 & $340 \cdot 8^{a, b}$ & 23.4 & $462 \cdot 1^{b}$ & 4.4 & NS & $<0.01$ & NS \\
\hline SFA $\ddagger$ & $1607 \cdot 2$ & 282.1 & $1307 \cdot 9$ & $16 \cdot 2$ & 1561.2 & 283.6 & $1725 \cdot 3$ & 78.4 & NS & NS & NS \\
\hline MUFA & 4548 & 906.1 & $3404 \cdot 8$ & $189 \cdot 4$ & $4376 \cdot 3$ & 797.9 & 4423.2 & $118 \cdot 2$ & NS & NS & NS \\
\hline PUFA & 1557.5 & 265.3 & $1490 \cdot 3$ & 81.3 & 2408.6 & 444.2 & 2906.6 & $120 \cdot 8$ & $<0.05$ & NS & NS \\
\hline LC-PUFA & $656 \cdot 4^{\mathrm{a}}$ & $95 \cdot 3$ & $738 \cdot 7^{\mathrm{a}}$ & $44 \cdot 3$ & $821 \cdot 9^{\mathrm{a}, \mathrm{b}}$ & 83.6 & $1072 \cdot 5^{\mathrm{b}}$ & $10 \cdot 3$ & $<0.05$ & NS & NS \\
\hline Trans & 33.1 & 6.6 & $22 \cdot 7$ & 0.9 & $25 \cdot 2$ & $6 \cdot 4$ & $24 \cdot 1$ & 0.4 & NS & NS & NS \\
\hline$n-6$ PUFA & $1074 \cdot 3$ & $196 \cdot 2$ & 841.8 & 50.1 & $1324 \cdot 6$ & 263.5 & 1377.0 & $50 \cdot 8$ & NS & NS & NS \\
\hline$n-6$ LC PUFA & 271.9 & $46 \cdot 6$ & 195.4 & $16 \cdot 6$ & 246.9 & 31.5 & 266.5 & 2.3 & NS & NS & NS \\
\hline$n-3$ PUFA & $446 \cdot 3^{\mathrm{a}}$ & $60 \cdot 1$ & $607 \cdot 2^{\mathrm{a}, \mathrm{b}}$ & 29 & $1056 \cdot 8^{\mathrm{b}, \mathrm{c}}$ & 175.6 & $1482^{c}$ & 68.8 & $<0.01$ & $<0.05$ & NS \\
\hline$n-3$ LC PUFA & $384.5^{\mathrm{a}}$ & 48.8 & $543 \cdot 2^{a}$ & $27 \cdot 7$ & $575 \cdot 1^{a}$ & 52.1 & $806 \cdot 0^{\mathrm{b}}$ & 8.0 & $<0.01$ & $<0.01$ & NS \\
\hline$n-6: n-3$ ratio & $2.4^{\mathrm{C}}$ & 0.1 & $1.4^{\mathrm{a}}$ & 0.0 & $1 \cdot 2^{\mathrm{a}, \mathrm{b}}$ & 0.0 & $0.9^{b}$ & 0.0 & $<0.001$ & $<0.001$ & $<0.01$ \\
\hline
\end{tabular}

LC, long chain.

* Treatment means were analysed by one-way ANOVA with Tukey's post hoc test of multiple comparisons. Effects of substrate, end product and substrate/end product interaction were analysed by two-way ANOVA.

* Treatment means were analysed by one-way AN
† See Table 1 for experimental diet abbreviations.

‡ See Table 2 for fatty acid classes and abbreviations. 


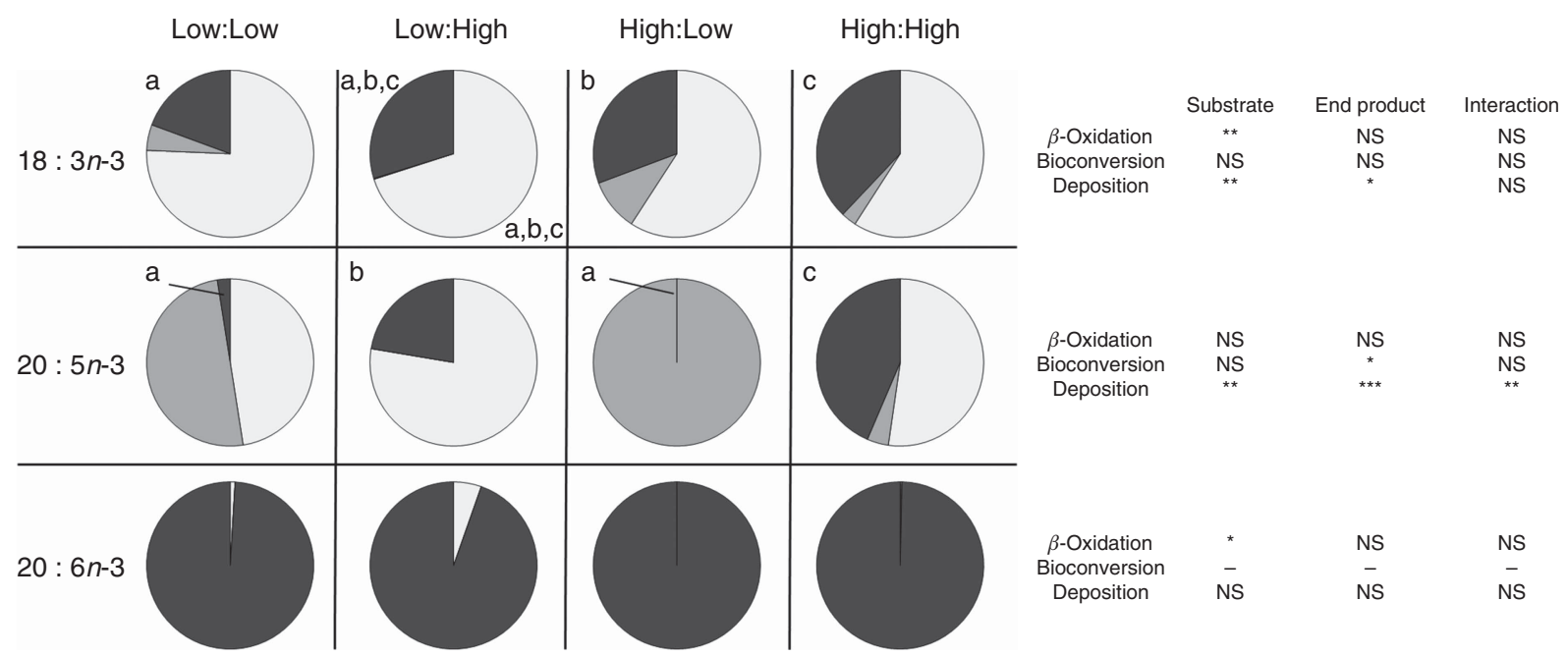

Fig. 1. Metabolic fate of dietary 18:3n-3, 20:5n-3 and $22: 6 n-3$ ( $\beta$-oxidation, bioconversion and deposition, expressed as percentage of net intake) in post-smolt Atlantic salmon fed experimental diets with altered dietary substrate $(18: 3 n-3)$ and end product $(20: 5 n-3$ and $22: 6 n-3)$ ratios for $283 \mathrm{~d}^{\text {a,b,c }}$ Values in the same row (either 18:3n-3, 20:5n-3 or $22: 6 n-3$ ) and for the same category ( $\beta$-oxidation, bioconversion and deposition) with unlike letters are significantly different $(P<0.05)$ and $P$ values relative to the two-way ANOVA comparing substrate, end product and substrate by end product interactions are reported on the right $\left({ }^{\star} P<0.05,{ }^{\star \star} P<0.01\right.$, $\left.{ }^{* * *} P<0.001\right)$. See Table 1 for experimental dietary abbreviations. $\square, \beta$-Oxidised (\%); $\square$, bioconverted (\%); $\square$, deposited (\%).

Apparent in vivo fatty acid bioconversion (expressed as nmol of fatty acid bioconverted per gram of fish per $\mathrm{d}$; $\mathrm{nmol} / \mathrm{g}$ per $\mathrm{d}$ and as percentage of net intake) (Table 7 and Fig. 1, respectively) highlighted the elongation of 18:0 in three of the four treatments (Low:Low, High:Low and High:High), with the highest values recorded in the High:Low treatment $(P<0 \cdot 05)$. There was a significant effect of end product concentration on the $\Delta-6$ desaturation of $18: 2 n-6(P<0 \cdot 001)$ with Low:Low recording the highest level of activity $(P<0 \cdot 05)$. Elongation of $18: 3 n-6$ was noted in all treatments; however, this was significantly higher in Low:Low $(P<0 \cdot 05)$. Likewise, $\Delta-5$ desaturation of $20: 3 n-6$ was highest in Low:Low $(P<0.05)$, whilst $\Delta-5$ desaturation of $20: 4 n-3$ was highest in High:Low, with negligible activity recorded in the other treatments $(P<0.05)$. The High:Low treatment recorded the highest level of $\Delta-6$ desaturation of $18: 3 n-3(P<0.05)$ and numerically, albeit not statistically, higher levels of $24: 6 n-3$ desaturation. In addition, there was a significant effect of substrate, end product and a substrate by end product interaction recorded for the $\Delta-6$ desaturation of $18: 3 n-3$. The High:Low treatment recorded significantly higher levels of elongation of $20: 5 n-3(P<0 \cdot 05)$ and also higher elongation of $22: 5 n-3$, though the results for the latter were not significant. Recorded levels of $24: 6 n-3$ chain shortening, the final step of endogenous $22: 6 n-3$ production, were highest in High:Low; despite relatively minimal amounts recorded in all other treatments, the differences were not significant $(P>0.05)$.

\section{Discussion}

While previous research has highlighted the capacity for endogenous $n$ - 3 LC PUFA production in salmonid species, the extent of this metabolic activity has, up until now, not been well quantified in post-smolt Atlantic salmon. In this respect, the present study demonstrated that the amount of apparent endogenous production of $n$-3 LC PUFA was variable between treatment groups of fish fed diets containing altered levels of dietary n-3 substrate, namely, $18: 3 n-3$, and end products, namely, $20: 5 n-3$ and $22: 6 n-3$. Despite this it was demonstrated that when provided a diet containing relatively high levels $18: 3 n-3$ and low levels of $20: 5 n-3$ and $22: 6 n-3$, post-smolt Atlantic salmon possesses a capacity for the endogenous synthesis of $n-3$ LC PUFA via in vivo fatty acid bioconversion. Moreover, when provided with abundant dietary supply of $18: 3 n-3$, the extent of endogenous production has the potential to enhance total fillet concentrations of $n-3$ LC PUFA, complementing, and independent of, dietary $n-3$ LC PUFA provision. Furthermore, the present study highlights the positive effect of high dietary substrate inclusion $(18: 3 n-3)$, concomitant with the supply of dietary fish oil, on the final concentration of fillet $n$-3 LC PUFA, attributable to the provision of a suitable substrate for $\beta$-oxidation, thus, sparing $n$ - 3 LC PUFA from catabolism. These important findings are discussed in further detail herein.

The effect of various lipid sources used in aquafeed on the nutritional value of the fish understandably garners substantial research attention. Nevertheless, growth performance remains a key indicator of the suitability of aquafeed formulations for the use in commercial aquaculture operations ${ }^{(60)}$. As reviewed by Glencross $^{(36)}$ and Turchini et al $^{(5)}$, the substitution of fish oil with terrestrial-based oils has been widely reported to have minimal effect on the growth of salmonids, even at high inclusion levels. Moreover, it has been reported that an excessive inclusion level of LC-PUFA may elicit detrimental effects on fish performance in several species ${ }^{(61-64)}$. However, currently used levels of dietary $n-3$ LC PUFA in commercial aquafeed are unlikely to elicit any negative effects on growth performance, given the low levels of marine-sourced oils presently utilised. Recent research advocates dietary $n$-3 LC PUFA at an inclusion level in excess of $2 \cdot 7 \%$ of fatty acids is necessary for optimal growth in post-smolt Atlantic salmon ${ }^{(10,59)}$. With respect to the 
Table 6. Apparent in vivo fatty acid $\beta$-oxidation (nmol/g per d) in Atlantic salmon fed the four experimental diets for $283 \mathrm{~d}^{\star}$ (Mean values with their standard errors, $n 2, N 8$ )

\begin{tabular}{|c|c|c|c|c|c|c|c|c|c|c|c|}
\hline & \multicolumn{8}{|c|}{ Diets† } & & & \\
\hline & \multicolumn{2}{|c|}{ Low:Low } & \multicolumn{2}{|c|}{ Low:High } & \multicolumn{2}{|c|}{ High:Low } & \multicolumn{2}{|c|}{ High:High } & \multicolumn{3}{|c|}{ Effect of substrate, end product and substrate/end product interaction ( $P$} \\
\hline & Mean & SEM & Mean & SEM & Mean & SEM & Mean & SEM & Substrate & End product & Interaction \\
\hline $12: 0$ & $2 \cdot 7^{\mathrm{a}}$ & 0.0 & $2 \cdot 8^{\mathrm{a}}$ & 0.1 & $1 \cdot 2^{\mathrm{b}}$ & 0.0 & $1.9^{\mathrm{b}}$ & 0.1 & $<0.001$ & $<0.05$ & NS \\
\hline $14: 0$ & $34 \cdot 1^{\mathrm{a}}$ & 0.5 & $60.7^{\mathrm{b}}$ & 0.4 & $18 \cdot 9^{\mathrm{c}}$ & 1.0 & $49 \cdot 3^{\mathrm{d}}$ & 3.5 & $<0.01$ & $<0.001$ & NS \\
\hline $16: 0$ & $526 \cdot 5^{a}$ & 2.9 & $537 \cdot 8^{\mathrm{a}}$ & $2 \cdot 7$ & $293 \cdot 8^{\mathrm{b}}$ & $2 \cdot 2$ & $367.5^{\mathrm{b}}$ & 23.4 & $<0.001$ & $<0.05$ & NS \\
\hline $18: 0$ & $121 \cdot 3^{a}$ & $2 \cdot 3$ & $130 \cdot 4^{a}$ & 0.4 & $42 \cdot 4^{b}$ & 5.7 & $85 \cdot 7^{b}$ & 6.4 & $<0.001$ & $<0.01$ & $<0.05$ \\
\hline $20: 0$ & - & & 5.0 & 0.0 & . & & & & - & - & - \\
\hline $22: 0$ & $1.0^{\mathrm{a}}$ & 0 & $2 \cdot 0^{\mathrm{b}}$ & 0.0 & $3.1^{c}$ & 0.2 & $4 \cdot 6^{d}$ & 0.2 & $<0.001$ & $<0.01$ & NS \\
\hline $24: 0$ & $1 \cdot 3^{a}$ & 0.1 & $2 \cdot 0^{b, c}$ & 0.1 & $2 \cdot 3^{\mathrm{c}}$ & 0.1 & $5 \cdot 3^{b}$ & 0.2 & $<0.001$ & $<0.001$ & $<0.01$ \\
\hline SFAß॥ & $686.9^{a}$ & 0.3 & $740 \cdot 7^{\mathrm{a}}$ & 2.7 & $361 \cdot 8^{\mathrm{b}}$ & 7.1 & $514 \cdot 3^{b}$ & 33.8 & $<0.001$ & $<0.01$ & $<0.05$ \\
\hline $14: 1 n-5$ & $5.9^{\mathrm{a}}$ & 0.1 & $5 \cdot 3^{\mathrm{b}}$ & 0.1 & $3 \cdot 2^{\mathrm{c}}$ & 0.0 & $2 \cdot 8^{\mathrm{C}}$ & 0.1 & $<0.001$ & $<0.01$ & NS \\
\hline $16: 1 n-7$ & $99.6^{\mathrm{a}}$ & 0.6 & $109.0^{\mathrm{a}}$ & 1.3 & $54.7^{b}$ & 2.5 & $71.0^{\mathrm{b}}$ & 6.5 & $<0.001$ & $<0.05$ & NS \\
\hline $18: 1 n-7$ & $26 \cdot 5$ & 1.0 & $30 \cdot 2$ & 1.0 & 21.5 & 0.2 & 28.0 & 3.5 & NS & NS & NS \\
\hline $18: 1 n-9$ & 556.0 & 38.3 & 551.6 & 11.4 & 382.5 & $12 \cdot 7$ & 371.6 & $56 \cdot 3$ & $<0.01$ & NS & NS \\
\hline $20: 1 n-9$ & - & & - & & 69.3 & 5.9 & 85.0 & $13 \cdot 1$ & - & - & - \\
\hline $22: 1 n-9$ & - & & - & & $15 \cdot 8^{a}$ & 1.3 & $25 \cdot 3^{\mathrm{b}}$ & 1.7 & - & - & - \\
\hline $24: 1 n-9$ & - & & $1 \cdot 2^{\mathrm{a}}$ & 0.2 & $1.9^{\mathrm{a}, \mathrm{b}}$ & 0.5 & $4.0^{\mathrm{a}}$ & 0.6 & $<0.01$ & $<0.05$ & NS \\
\hline $20: 1 n-11$ & - & & 3.5 & 0.1 & 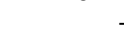 & & & & - & - & - \\
\hline $22: 1 n-11$ & $3.6^{a}$ & 0.1 & $6.0^{\mathrm{b}}$ & 0.2 & $3.0^{\mathrm{a}}$ & 0.0 & $5 \cdot 8^{\mathrm{b}}$ & 0.5 & NS & $<0.01$ & NS \\
\hline MUFA & 691.6 & 40.1 & $706 \cdot 7$ & 14.1 & 551.9 & $18 \cdot 2$ & 593.6 & 82.5 & NS & NS & NS \\
\hline $18: 2 n-6$ & 179.2 & 8.6 & $170 \cdot 8$ & 0.2 & $203 \cdot 1$ & $12 \cdot 2$ & 197.8 & $22 \cdot 1$ & NS & NS & NS \\
\hline $20: 2 n-6$ & - & & - & & 1.0 & 1.0 & 1.9 & 1.9 & - & - & - \\
\hline $22: 2 n-6$ & - & & - & & 0.4 & 0.1 & 0.5 & 0.2 & - & - & - \\
\hline $20: 4 n-6$ & - & & 1.7 & 0.3 & . & & 2.4 & 0.8 & - & - & - \\
\hline $22: 4 n-6$ & - & & $0.4^{\mathrm{a}}$ & 0.0 & $0.1^{\mathrm{a}}$ & 0.1 & $0.6^{\mathrm{b}}$ & 0.1 & NS & $<0.01$ & NS \\
\hline $22: 5 n-6$ & - & & $1.5^{\mathrm{a}}$ & 0.1 & $0.1^{\mathrm{b}}$ & 0.1 & $1.7^{\mathrm{a}}$ & 0.2 & NS & $<0.001$ & NS \\
\hline$n-6$ PUFA & $179 \cdot 2$ & 8.6 & 174.4 & 0.1 & 204.7 & 13.1 & $204 \cdot 8$ & 23.6 & NS & NS & NS \\
\hline $18: 3 n-3$ & $50 \cdot 6^{a}$ & 2.6 & $41.9^{a}$ & 0.1 & $251 \cdot 6^{\mathrm{b}}$ & 0.2 & $273.5^{\mathrm{b}}$ & 16.6 & $<0.001$ & NS & NS \\
\hline $18: 4 n-3$ & 0.9 & 0.9 & 1.3 & 0.0 & . & & & & - & - & - \\
\hline $20: 4 n-3$ & 0.3 & 0.3 & 1.2 & 0.1 & . & & & & - & - & - \\
\hline $20: 3 n-3$ & 0.1 & 0.1 & - & & 3.0 & 0.8 & 1.0 & 1.0 & $<0.05$ & NS & NS \\
\hline $22: 3 n-3$ & - & & - & & 2.6 & 0.1 & $2 \cdot 8$ & 0.2 & - & - & - \\
\hline $20: 5 n-3$ & $5 \cdot 7$ & $5 \cdot 7$ & $42 \cdot 3$ & 0.1 & . & & 28.0 & 1.7 & $<0.05$ & $<0.001$ & NS \\
\hline $22: 5 n-3$ & 1.8 & 1.8 & 4.3 & 0.1 & . & & & & - & - & - \\
\hline $22: 6 n-3$ & 0.2 & 0.2 & 3.2 & 1.0 & . & & 0.2 & 0.2 & $<0.05$ & $<0.05$ & NS \\
\hline$n-3$ PUFA & $59 \cdot 4^{\mathrm{a}}$ & 11.3 & $94 \cdot 2^{a}$ & 1.5 & $257 \cdot 2^{\mathrm{b}}$ & $1 \cdot 1$ & $305.5^{\mathrm{b}}$ & 15.8 & $<0.001$ & $<0.05$ & NS \\
\hline Total FA & 2549.7 & $75 \cdot 2$ & $2700 \cdot 3$ & 33.7 & 2394.7 & $72 \cdot 1$ & $2731 \cdot 8$ & 278 & NS & NS & NS \\
\hline
\end{tabular}

a,b,c,d Mean values within a row with unlike superscript letters were significantly different $(P<0.05)$.

*Treatment means were analysed by one-way ANOVA with Tukey's post hoc test of multiple comparisons. Effects of substrate, end product and substrate/end product interaction were analysed by two-way ANOVA. † See Table 1 for experimental diet abbreviations.

$\neq \beta$-Oxidation not detected.

$\S$ See Table 2 for fatty acid classes and abbreviations.

॥I Fatty acids not recording any $\beta$-oxidation are not reported in this table. 
Table 7. Apparent in vivo fatty acid bioconversion (nmol/g per d) in Atlantic salmon fed the four experimental diets for $283 \mathrm{~d}^{*}$ (Mean values with their standard errors, $n 2, N 8$ )

\begin{tabular}{|c|c|c|c|c|c|c|c|c|c|c|c|}
\hline & \multicolumn{8}{|c|}{ Diets† } & & & \\
\hline & \multicolumn{2}{|c|}{ Low:Low } & \multicolumn{2}{|c|}{ Low:High } & \multicolumn{2}{|c|}{ High:Low } & \multicolumn{2}{|c|}{ High:High } & \multicolumn{3}{|c|}{ Effect of substrate, end product and substrate/end product interaction $(P$} \\
\hline & Mean & SEM & Mean & SEM & Mean & SEM & Mean & SEM & Substrate & End product & Interaction \\
\hline \multicolumn{12}{|l|}{ Fatty acid elongation $\ddagger$} \\
\hline $18: 0$ to $20: 0$ & $1 \cdot 28^{\mathrm{a}}$ & 0.06 & - & & $31.56^{\mathrm{b}}$ & $5 \cdot 72$ & $9.83^{\mathrm{a}}$ & $1 \cdot 27$ & $<0.01$ & $<0.05$ & $<0.05$ \\
\hline $18: 1 n-9$ to $20: 1 n-9$ & $20 \cdot 15$ & 4.38 & 10.68 & 2.25 & \multicolumn{2}{|c|}{ - } & \multicolumn{2}{|c|}{ - } & - & - & - \\
\hline $20: 1 n-9$ to $22: 1 n-9$ & 2.06 & 0.60 & 0.25 & 0.24 & \multicolumn{2}{|c|}{-} & \multicolumn{2}{|c|}{-} & - & - & - \\
\hline $22: 1 n-9$ to $24: 1 n-9$ & 0.27 & 0.07 & - & & \multicolumn{2}{|c|}{ - } & \multicolumn{2}{|c|}{ - } & - & - & - \\
\hline $18: 2 n-6$ to $20: 2 n-6$ & $10 \cdot 31^{\mathrm{a}}$ & 1.87 & $10 \cdot 82^{\mathrm{a}}$ & 0.80 & $0.26^{\mathrm{b}}$ & 0.26 & $0.55^{\mathrm{b}}$ & 0.55 & $<0.01$ & NS & NS \\
\hline $20: 2 n-6$ to $22: 2 n-6$ & 0.85 & 0.13 & 0.92 & 0.07 & - & & \multicolumn{2}{|c|}{-} & - & - & - \\
\hline $18: 3 n-6$ to $20: 3 n-6$ & $39 \cdot 18^{\mathrm{a}}$ & 5.31 & $9 \cdot 84^{\mathrm{b}}$ & 0.77 & $21 \cdot 19^{b}$ & 0.77 & $8 \cdot 71^{\mathrm{b}}$ & $1 \cdot 10$ & $<0.05$ & $<0.01$ & $<0.05$ \\
\hline $20: 4 n-6$ to $22: 4 n-6$ & $2 \cdot 31$ & 0.11 & \multirow{2}{*}{\multicolumn{2}{|c|}{-}} & 0.08 & 0.08 & \multicolumn{2}{|c|}{ - } & - & - & - \\
\hline $22: 4 n-6$ to $24: 4 n-6$ & 1.73 & 0.11 & & & 0.03 & 0.03 & \multicolumn{2}{|c|}{-} & - & - & - \\
\hline $18: 3 n-3$ to $20: 3 n-3$ & 0.02 & 0.02 & 0.10 & 0.10 & - & & 0.50 & 0.50 & NS & NS & NS \\
\hline $18: 4 n-3$ to $20: 4 n-3$ & $4 \cdot 23^{\mathrm{a}}$ & $4 \cdot 23$ & \multicolumn{2}{|c|}{ - } & $43 \cdot 64^{b}$ & 6.44 & $13.48^{\mathrm{a}}$ & 0.33 & $<0.01$ & $<0.05$ & $<0.05$ \\
\hline $20: 5 n-3$ to $22: 5 n-3$ & $8 \cdot 10^{\mathrm{a}}$ & $8 \cdot 10$ & \multicolumn{2}{|c|}{-} & $24 \cdot 82^{b}$ & $5 \cdot 51$ & $2 \cdot 22^{\mathrm{a}}$ & 1.02 & NS & $<0.05$ & NS \\
\hline $22: 5 n-3$ to $24: 5 n-3$ & 8.58 & 8.53 & 0.36 & 0.09 & $20 \cdot 76$ & 4.19 & 1.85 & 0.80 & NS & $<0.05$ & NS \\
\hline \multicolumn{12}{|c|}{ Fatty acid $\Delta-9$ desaturation } \\
\hline $20: 0$ to $20: 1 n-11$ & $3.67^{\mathrm{a}}$ & 0.13 & \multicolumn{2}{|c|}{-} & $43 \cdot 24^{\mathrm{b}}$ & $5 \cdot 13$ & \multicolumn{2}{|l|}{$26 \cdot 37^{\mathrm{c}}$} & $<0.001$ & $<0.05$ & NS \\
\hline \multicolumn{12}{|c|}{ Fatty acid $\Delta-6$ desaturation } \\
\hline $18: 2 n-6$ to $18: 3 n-6$ & $52 \cdot 74^{\mathrm{a}}$ & 4.75 & $13 \cdot 28^{b}$ & 0.69 & $36.79^{\mathrm{C}}$ & $1 \cdot 12$ & $15 \cdot 26^{\mathrm{b}, \mathrm{c}}$ & $1 \cdot 31$ & NS & $<0.001$ & $<0.05$ \\
\hline $24: 4 n-6$ to $24: 5 n-6$ & 1.73 & 0.11 & \multicolumn{2}{|c|}{ - } & 0.03 & 0.03 & \multicolumn{2}{|c|}{ - } & - & - & - \\
\hline $18: 3 n-3$ to $18: 4 n-3$ & $3 \cdot 31^{\mathrm{a}}$ & 3.31 & - & & $43.02^{b}$ & $6 \cdot 52$ & $13 \cdot 16^{\mathrm{a}}$ & 0.33 & $<0.01$ & $<0.05$ & $<0.05$ \\
\hline $24: 5 n-3$ to $24: 6 n-3$ & 8.59 & 8.45 & 0.38 & 0.04 & $20 \cdot 40$ & $4 \cdot 16$ & 1.52 & 0.91 & NS & $<0.05$ & NS \\
\hline Fatty acid $\Delta-5$ desaturat & & & & & & & & & & & \\
\hline $20: 3 n-6$ to $20: 4 n-6$ & $15 \cdot 91^{a}$ & $2 \cdot 61$ & - & & $5.07^{b}$ & 0.41 & - & & - & - & - \\
\hline $20: 4 n-3$ to $20: 5 n-3$ & $4.00^{\mathrm{a}}$ & 4.00 & - & & $31.50^{\mathrm{b}}$ & $6 \cdot 16$ & - & & - & - & - \\
\hline Fatty acid chain shorten & & & & & & & & & & & \\
\hline $24: 5 n-6$ to $22: 5 n-6$ & $1.73^{\mathrm{a}}$ & 0.11 & - & & $0.03^{\mathrm{b}}$ & 0.03 & - & & - & - & - \\
\hline $24: 6 n-3$ to $22: 6 n-3$ & 8.45 & 8.45 & - & & 19.89 & 4.26 & 1.02 & 1.02 & NS & $<0.05$ & NS \\
\hline
\end{tabular}
a,b,c Mean values within a row with unlike superscript letters were significantly different $(P<0.5)$.
* Treatment means were analysed by one-way ANOVA with Tukey's post hoc test of multiple comparisons. Effects of substrate, end product and substrate/end product interaction were analysed by two-way ANOVA. † See Table 1 for experimental diet abbreviations.

Fatly acids not recording any bioconversion (elongation or desaturation) are not reported in this table.

$\S$ Not detected. 
present study, there was no difference between major growth parameters between any of the treatments, despite no added fish oil in two of the four dietary treatments; hence, it cannot support this minimum recommended requirement for dietary $n$-3 LC PUFA in terms of growth performance.

Despite apparent contention regarding growth performance, it is widely accepted that the amount of $n-3$ LC PUFA in the fillet is a reflection of the dietary inclusion level, as reviewed by Bendiksen et $a l^{(1)}$, Tocher ${ }^{(2)}$, Turchini et $a l^{(5)}$ and Sales \& Glencross $^{(65)}$. However, given the demonstrated capacity of Atlantic salmon to endogenously synthesise 22:6n-3 from dietary precursors ${ }^{(59,66)}$, the mirroring effect between diet and fillet fatty acid compositions may be partially obscured, especially when diets are devoid of added fish oil ${ }^{(2,67)}$. Despite this, the biosynthesis of $n-3$ LC PUFA from $18: 3 n-3$ in salmonids is recognised as a mechanism to satiate the fundamental physiological minimal requirements of $n-3$ LC PUFA and is generally insufficient to enrich fillets with $n$ - 3 LC PUFA to the same extent as dietary added fish oil and in line with consumer expectations ${ }^{(5,68)}$. Therefore, the numerically higher fillet levels of $n-3$ LC PUFA, expressed as both $\mu \mathrm{mol} / \mathrm{g}$ and $\mathrm{mg} / 100 \mathrm{~g}$ of fillet tissue recorded in fish fed the High:Low diet compared with the Low: High diet, were somewhat unexpected considering the High: Low diet contained no added fish oil. Although it should be noted that similar results were not observed between fish fed the High:Low diet and fish fed the High:High diet. Nevertheless, as it will be discussed, these results highlight differences in lipid metabolism, namely, $\beta$-oxidation, bioconversion and deposition of dietary fatty acids, specifically, $18: 3 n-3,20: 5 n-3$ and $22: 6 n-3$.

It is well understood that dietary fatty acids provide the vast majority of metabolic energy for salmonids ${ }^{(12)}$. In particular, MUFA, including 18:1n-9, and SFA, including 16:0, are known to be readily catabolised for this specific purpose ${ }^{(69,70)}$. However, due to the increasing commercial utilisation of dietary oils rich in $\mathrm{C}_{18} n$-3 PUFA in aquafeed formulations (e.g. canola oil, linseed oil and potentially in the future, camelina oil) $18: 3 n-3$ is increasingly relied upon by farmed salmon as a dietary energy substrate ${ }^{(10,40,71,72)}$. Research suggests $18: 3 n-3$ is readily catabolised in fish, including salmonids, proportional to dietary inclusion levels ${ }^{(30,73-75)}$. With respect to the present study, dietary $n$-3 PUFA substrate level had a significant effect on the $\beta$-oxidation of $18: 3 n-3 \quad(P<0 \cdot 001)$. Specifically, an increase in dietary $18: 3 n-3$ led to a proportional decrease in $\beta$-oxidation of $18: 3 n-3$ (in terms of percentage of net intake). However, considering the enhanced dietary supply of $18: 3 n-3$ in the high-substrate treatments, quantitatively more $18: 3 n-3$ was $\beta$-oxidised in both high dietary $18: 3 n-3$ treatments (in terms of $\mathrm{nmol} / \mathrm{g}$ per $\mathrm{d}$ ). Furthermore, the deposition of $18: 3 n-3$ proportionally increased with an increased dietary supply. This is in contrast to the notion of decreasing deposition efficiency of $n$-3 PUFA with increased dietary supply ${ }^{(51,73,76,77)}$. However, considering the increased total amount of $18: 3 n-3$ in the high dietary $18: 3 n-3$ treatments, the actual amount of $\beta$-oxidised was greater than the low dietary $18: 3 n-3$ treatments.

In addition to providing a suitable substrate for $\beta$-oxidation in salmonids, the first step of $n-3$ PUFA bioconversion has been shown to be primarily modulated by the availability of $\mathrm{C}_{18} n-3$ PUFA by providing a substrate for $\Delta-6$ desaturase activity ${ }^{(10,31,32,34,41)}$. The findings of the present study support this, and it was found that the $\Delta-6$ desaturation of $18: 3 n-3$ was significantly enhanced by the dietary addition of $18: 3 n-3$ (in terms of both nmol/g per $\mathrm{d}$ and percentage of net intake). In addition, and in accordance with previous research, the absence of added dietary n-3 LC PUFA appeared to further enhance bioconversion activity relative to the high dietary $n-3$ LC PUFA treatments owing to the absence of a previously identified negative feedback mechanism acting upon the $n-3$ LC PUFA bioconversion pathway in both mice and Atlantic salmon $^{(39,78)}$.

Further to $18: 3 n-3$ bioconversion, there were observable differences between treatments in relation to the metabolism of $20: 5 n-3$. Previous research has suggested $n-3$ LC PUFA can be preserved from catabolism by careful manipulation of the dietary fatty acid composition termed ' $n-3$ LC PUFA sparing,(74,79-82). Specifically, the dietary addition of saturated and MUFA has been shown to enhance the retention efficiency of $n$ - 3 LC PUFA $^{(6,51,74)}$. With respect to the present study, diet had a clear effect on the deposition of $20: 5 n-3$. The Low:High treatment appeared to favour the catabolism of longer, more unsaturated fatty acids, namely, 20:5n-3. In contrast, the High:High treatment had a lesser reliance on $n$ - 3 LC PUFA $\beta$-oxidation and instead appeared to preferentially $\beta$-oxidise $18: 3 n-3$. These findings support previous research advocating the suitability of $18: 3 n-3$ as a dietary energy substrate ${ }^{(22,72,83-85)}$. Moreover, they agree with the findings in juvenile salmon advocating the potential for added dietary $18: 3 n$ - 3 to spare $n$ - 3 LC PUFA from catabolism ${ }^{(86)}$.

Despite a very limited dietary provision of $n$ - 3 LC PUFA in the High:Low treatment, virtually all endogenously synthesised $20: 5 n$-3 was further bioconverted, culminating in a recorded chain-shortening of $24: 6 n-3$ - the final step in $n-3$ LC PUFA biosynthesis - to produce 22:6n-3. In accordance with previous research, it therefore appears that a production of $22: 6 n-3$ was favoured over $20: 5 n-3$ deposition, which appears to be present predominately as an intermediate step toward the de novo biosynthesis of $22: 6 n-3^{(46,73,87)}$. Furthermore, in accordance with previous research that identified a negative feedback mechanism on the $n$-3 PUFA bioconversion pathway, the presence of dietary $n-3$ LC PUFA appeared to negatively affect the bioconversion of $20: 5 n-3^{(12,33,78)}$.

In general, dietary $22: 6 n-3$ is well conserved from catabolism in Atlantic salmon, resulting in similar fillet levels to those provided by the $\operatorname{diet}^{(9,75,84,88,89)}$. The present study largely confirms this and high retention of $22: 6 n-3$ was recorded in all treatments. However, a significant effect of dietary substrate level $(18: 3 n-3)$ on the $\beta$-oxidation of $22: 6 n-3$ was recorded. Specifically, the $\beta$-oxidation of $22: 6 n-3$ was reduced in both high dietary substrate $(18: 3 n-3)$ treatments. Resultantly, a high dietary 18:3n-3 content provided (i) a suitable and highly available $\beta$-oxidation substrate that, consequently, enabled the sparing of $n$ - 3 LC PUFA from $\beta$-oxidation, (ii) an enhanced substrate for bioconversion and (iii) in contrast to high end product treatments, it did not inhibit $n-3$ LC PUFA biosynthesis which, as mentioned, can elicit a negative feedback mechanism on the $n$-3 PUFA bioconversion pathway ${ }^{(12,78)}$. Consequently, 
the addition of abundant dietary $18: 3 n-3$ in diets devoid of added fish oil had a positive effect on the final n-3 LC PUFA level in the fillet of post-smolt Atlantic salmon.

In the absence of added fish oil, the high dietary provision of $18: 3 n-3$ potentially provided multiplicative benefits in terms of facilitating the deposition of $n-3$ LC PUFA in to the fillet tissue by favouring the $\beta$-oxidation of dietary $\mathrm{C}_{18} n-3$ PUFA resulting in an apparent conservation of $n-3$ LC PUFA from catabolism and by providing sufficient substrate for $n-3$ LC PUFA biosynthesis. These findings demonstrate the capacity for endogenous $n$-3 LC PUFA synthesis in post-smolt Atlantic salmon to ameliorate the potential negative consequences on fillet nutritional quality often attributed to a severe reduction or removal of dietary provided fish oil. This provides a commercially relevant platform for the further development of tailored diets with respect to production stage to ensure Atlantic salmon is highly nutritious at the time of harvest. Complementary methods, such as real-time PCR and stable isotope analysis, are suggested in future trials on postsmolt Atlantic salmon. This will enable a better quantification of the possible modulation of known genes associated with n-3 LC PUFA bioconversion processes and a more accurate trace of individual fatty acids along the $n-3$ LC PUFA bioconversion pathway, and in particular the ability to differentiate from de novo synthesised LC-PUFA $v$. LC-PUFA spared from catabolic processes. Nevertheless, the information provided herein, scant for post-smolt Atlantic salmon, is essential to create dietary strategies that mitigate a 'low fish oil future'.

\section{Acknowledgements}

The authors gratefully acknowledge the assistance and support from Ridley Aquafeed (Brisbane, Australia). The authors also wish to thank Dr Karen Hermon, Mr Robert Collins and Ms Zoe Robertson for technical assistance and valuable contribution to this study.

The authors wish to declare funding and provision of dietary ingredients from Ridley Aquafeed (Brisbane, Australia) under the Deakin-Ridley Aquaculture Research Initiative.

T. S. M. conducted the experiment and subsequent analysis and wrote the manuscript. D. S. F. contributed to the concept and design of the experiment and critically revised the manuscript. M. K. J. assisted in conducting the experiment and subsequent analysis. B. D. G. contributed to the concept and design of the experiment and critically revised the manuscript. R. P. S. contributed to the concept and design of the experiment and critically revised the manuscript. G. M. T. contributed to the concept and design of the experiment and critically revised the manuscript.

The authors wish to declare funding and donation of materials for the growth trial from Ridley Aquafeed Ltd (Brisbane, Australia). The authors wish to clarify that the present trial compared experimental dietary lipid compositions in aquafeed and was not comparing commercially produced products by Ridley Aquafeed Ltd.

\section{References}

1. Bendiksen EÅ, Johnsen CA, Olsen HJ, et al. (2011) Sustainable aquafeeds: progress towards reduced reliance upon marine ingredients in diets for farmed Atlantic salmon (Salmo salar L.). Aquaculture 314, 132-139.

2. Tocher DR. (2015) Omega-3 long-chain polyunsaturated fatty acids and aquaculture in perspective. Aquaculture $\mathbf{4 4 9}$, 94-107.

3. Turchini GM, Ng W-K \& Tocher DR. (2010) Fish Oil Replacement and Alternative Lipid Sources in Aquaculture Feeds. Boca Raton, FL: CRC Press.

4. Sprague M, Dick JR \& Tocher DR. (2016) Impact of sustainable feeds on omega-3 long-chain fatty acid levels in farmed Atlantic salmon, 2006-2015. Sci Rep 6, 21892.

5. Turchini GM, Torstensen BE \& Ng W-K. (2009) Fish oil replacement in finfish nutrition. Rev Aquac 1, 10-57.

6. Emery JA, Smullen RP, Keast RSJ, et al. (2016) Viability of tallow inclusion in Atlantic salmon diet, as assessed by an onfarm grow out trial. Aquaculture 451, 289-297.

7. Francis DS \& Turchini GM. (2017) Retro-engineering the protein sparing effect to preserve $n$ - 3 LC-PUFA from catabolism and optimise fish oil utilisation: a preliminary case study on juvenile Atlantic salmon. Aquaculture $\mathbf{4 6 8}$, 184-192.

8. Nuez Ortin W, Carter C, Nichols P, et al. (2015) Increasing deposition efficiency of $n$-3 LC-PUFA in Altlantic salmon smolt using high DHA and ALA oils. 2015 Aquaculture Europe, 20-23 October, Rotterdam, Holland (conference edited).

9. Torstensen BE, Frøyland L \& Lie Ø. (2004) Replacing dietary fish oil with increasing levels of rapeseed oil and olive oil effects on Atlantic salmon (Salmo salar L.) tissue and lipoprotein lipid composition and lipogenic enzyme activities. Aquacult Nutr 10, 175-192.

10. Hixson SM, Parrish CC, Xue X, et al. (2017)) Growth performance, tissue composition, and gene expression responses in Atlantic salmon (Salmo salar) fed varying levels of different lipid sources. Aquaculture 467, 76-88.

11. Norambuena F, Morais S, Emery JA, et al. (2015) Arachidonic acid and eicosapentaenoic acid metabolism in juvenile Atlantic salmon as affected by water temperature. PLOS ONE 10, e0143622.

12. Tocher DR. (2003) Metabolism and functions of lipids and fatty acids in teleost fish. Rev Fish Sci 11, 107-184.

13. Torstensen BE, Lie $\varnothing$ \& Frøyland L. (2000) Lipid metabolism and tissue composition in Atlantic salmon (Salmo salar L.) effects of capelin oil, palm oil, and oleic acid-enriched sunflower oil as dietary lipid sources. Lipids 35, 653-664.

14. Karalazos V, Bendiksen EÅ, Dick JR, et al. (2011) Influence of the dietary protein: lipid ratio and fish oil substitution on fatty acid composition and metabolism of Atlantic salmon (Salmo salar) reared at high water temperatures. Br J Nutr $\mathbf{1 0 5}$, 1012-1025.

15. Monroig Ó, Zheng X, Morais S, et al. (2010) Multiple genes for functional $\Delta 6$ fatty acyl desaturases (Fad) in Atlantic salmon (Salmo salar L.): gene and cDNA characterization, functional expression, tissue distribution and nutritional regulation. BiOchim Biophys Acta 1801, 1072-1081.

16. Nakamura MT \& Nara TY (2004) Structure, function, and dietary regulation of $\Delta 6, \Delta 5$, and $\Delta 9$ desaturases. Nutrition 24, 345-376.

17. Castro LFC, Tocher DR \& Monroig Ó. (2016) Long-chain polyunsaturated fatty acid biosynthesis in chordates: insights into the evolution of Fads and Elovl gene repertoire. Prog Lipid Res 62, 25-40. 
18. Monroig Ó, Webb K, Ibarra-Castro L, et al. (2011) Biosynthesis of long-chain polyunsaturated fatty acids in marine fish: characterization of an Elovl4-like elongase from cobia Rachycentron canadum and activation of the pathway during early life stages. Aquaculture 312, 145-153.

19. Morais S, Monroig Ó, Zheng X, et al. (2009) Highly unsaturated fatty acid synthesis in atlantic salmon: characterization of ELOVL5-and ELOVL2-like elongases. Mar Biotechnol 11, 627-639.

20. Venegas-Calerón M, Sayanova O \& Napier JA. (2010) An alternative to fish oils: metabolic engineering of oil-seed crops to produce omega-3 long chain polyunsaturated fatty acids. Prog Lipid Res 49, 108-119.

21. Kabeya N, Fonseca MM, Ferrier DE, et al. (2018) Genes for de novo biosynthesis of omega-3 polyunsaturated fatty acids are widespread in animals. Sci Adv 4, eaar6849.

22. Bell JG, McEvoy J, Tocher DR, et al. (2001) Replacement of fish oil with rapeseed oil in diets of Atlantic salmon (Salmo salar) affects tissue lipid compositions and hepatocyte fatty acid metabolism. J Nutr 131, 1535-1543.

23. Ruyter B \& Thomassen MS (1999) Metabolism of $n-3$ and $n$-6 fatty acids in Atlantic salmon liver: stimulation by essential fatty acid deficiency. Lipids 34, 1167-1176.

24. Sissener N, Sanden M, Torstensen B, et al. (2016) High dietary 18: $2 n-6 / 18: 3 n-3$ ratio does not inhibit elongation and desaturation of 18:3n-3 to EPA and DHA in Atlantic salmon (Salmo salar L.). Aquacult Nutr 23, 899-909.

25. Turchini G, Hermon K, Cleveland B, et al. (2013) Seven fish oil substitutes over a rainbow trout grow-out cycle: I) Effects on performance and fatty acid metabolism. Aquacult Nutr 19, $82-94$.

26. Tocher D, Bell JG, Dick J, et al. (1997) Fatty acyl desaturation in isolated hepatocytes from Atlantic salmon (Salmo salar): stimulation by dietary borage oil containing $\gamma$-linolenic acid. Lipids 32, 1237-1247.

27. Tocher DR, Bell JG, Dick JR, et al. (2000) Polyunsaturated fatty acid metabolism in Atlantic salmon (Salmo salar) undergoing parr-smolt transformation and the effects of dietary linseed and rapeseed oils. Fish Physiol Biochem 23, 59-73.

28. Giri SS, Graham J, Hamid NKA, et al. (2016) Dietary micronutrients and in vivo $n$-3 LC-PUFA biosynthesis in Atlantic salmon. Aquaculture 452, Suppl. C, 416-425.

29. Nordgarden U, Torstensen BE, Frøyland L, et al. (2003) Seasonally changing metabolism in Atlantic salmon (Salmo salar $\mathrm{L}$.) II $-\beta$-oxidation capacity and fatty acid composition in muscle tissues and plasma lipoproteins. Aquacult Nutr $\mathbf{9}$, 295-303.

30. Tocher D, Fonseca-Madrigal J, Bell JG, et al. (2002) Effects of diets containing linseed oil on fatty acid desaturation and oxidation in hepatocytes and intestinal enterocytes in Atlantic salmon (Salmo salar). Fish Physiol Biochem 26, 157-170.

31. Glencross B, Tocher D, Matthew C, et al. (2014) Interactions between dietary docosahexaenoic acid and other long-chain polyunsaturated fatty acids on performance and fatty acid retention in post-smolt Atlantic salmon (Salmo salar). Fish Physiol Biochem 40, 1213-1227.

32. Thanuthong T, Francis DS, Manickam E, et al. (2011) Fish oil replacement in rainbow trout diets and total dietary PUFA content: II) Effects on fatty acid metabolism and in vivo fatty acid bioconversion. Aquaculture 322-323, 99-108.

33. Thomassen MS, Rein D, Berge GM, et al. (2012) High dietary EPA does not inhibit Delta 5 and Delta 6 desaturases in Atlantic salmon (Salmo salar L.) fed rapeseed oil diets. Aquaculture 360, 78-85.
34. Turchini GM \& Francis DS. (2009) Fatty acid metabolism (desaturation, elongation and $\beta$-oxidation) in rainbow trout fed fish oil- or linseed oil-based diets. Br J Nutr 102, 69-81.

35. Mellery J, Geay F, Tocher DR, et al. (2016) Temperature increase negatively affects the fatty acid bioconversion capacity of rainbow trout (Oncorbynchus mykiss) fed a linseed oilbased diet. PLOS ONE 11, e0164478.

36. Glencross BD. (2009) Exploring the nutritional demand for essential fatty acids by aquaculture species. Rev Aquac 1, 71-124.

37. Kjær MA, Ruyter B, Berge GM, et al. (2016) Regulation of the omega-3 fatty acid biosynthetic pathway in Atlantic salmon hepatocytes. PLOS ONE 11, e 0168230.

38. Leaver MJ, Villeneuve LAN, Obach A, et al. (2008) Functional genomics reveals increases in cholesterol biosynthetic genes and highly unsaturated fatty acid biosynthesis after dietary substitution of fish oil with vegetable oils in Atlantic salmon (Salmo salar). BMC Genomics 9, 299.

39. Tocher DR, Bell JG, Dick JR, et al. (2003) Effects of dietary vegetable oil on Atlantic salmon hepatocyte fatty acid desaturation and liver fatty acid compositions. Lipids 38, 723-732.

40. Turchini GM, Nichols PD, Barrow C, et al. (2011) Jumping on the omega- 3 bandwagon: distinguishing the role of long-chain and short-chain omega-3 fatty acids. Crit Rev Food Sci Nutr 52, 795-803.

41. Xue X, Hixson SM, Hori TS, et al. (2015) Atlantic salmon (Salmo salar) liver transcriptome response to diets containing Camelina sativa products. Comp Biochem Physiol Part D Genomics Proteomics 14, 1-15.

42. Lewis MJ, Hamid NKA, Alhazzaa R, et al. (2013) Targeted dietary micronutrient fortification modulates $n-3$ LC-PUFA pathway activity in rainbow trout (Oncorbynchus mykiss). Aquaculture 412, 215-222.

43. Senadheera SD, Turchini GM, Thanuthong T, et al. (2012) Effects of dietary iron supplementation on growth performance, fatty acid composition and fatty acid metabolism in rainbow trout (Oncorbynchus mykiss) fed vegetable oil based diets. Aquaculture 342, 80-88.

44. Senadheera SD, Turchini GM, Thanuthong T, et al. (2012) Effects of dietary vitamin $\mathrm{B}_{6}$ supplementation on fillet fatty acid composition and fatty acid metabolism of rainbow trout fed vegetable oil based diets. I Agric Food Chem 60, 2343-2353.

45. Zheng X, Torstensen BE, Tocher DR, et al. (2005) Environmental and dietary influences on highly unsaturated fatty acid biosynthesis and expression of fatty acyl desaturase and elongase genes in liver of Atlantic salmon (Salmo salar). Biochim Biophys Acta 1734, 13-24.

46. Tocher DR. (2010) Fatty acid requirements in ontogeny of marine and freshwater fish. Aquac Res 41, 717-732.

47. Christenson JK, O'Kane GM, Farmery AK, et al. (2017) The barriers and drivers of seafood consumption in Australia: a narrative literature review. Int J Consum Stud 41, 299-311.

48. Strobel C, Jahreis G \& Kuhnt K (2012) Survey of $n-3$ and $n-6$ polyunsaturated fatty acids in fish and fish products. Lipids Health Dis 11, 144

49. Tacon AGJ \& Metian M (2015) Feed matters: satisfying the feed demand of aquaculture. Rev Fish Sci Aquac 23, 1-10.

50. Talbot C, Corneillie S \& Korsøen $\varnothing$ (1999) Pattern of feed intake in four species of fish under commercial farming conditions: implications for feeding management. Aquac Res 30, 509-518.

51. Francis DS, Thanuthong T, Senadheera SPSD, et al. (2014) n-3 LC-PUFA deposition efficiency and appetite-regulating hormones are modulated by the dietary lipid source during 
rainbow trout grow-out and finishing periods. Fish Physiol Biochem 40, 577-593.

52. Emery JA, Hermon K, Hamid NK, et al. (2013) $\Delta-6$ desaturase substrate competition: dietary linoleic acid $(18: 2 n-6)$ has only trivial effects on $\alpha$-linolenic acid (18:3n-3) bioconversion in the teleost rainbow trout. PLOS ONE 8, e57463.

53. Folch J, Lees M \& Sloane-Stanley G (1957) A simple method for the isolation and purification of total lipids from animal tissues. J Biol Chem 226, 497-509.

54. Norambuena F, Lewis M, Hamid NKA, et al. (2013) Fish oil replacement in current aquaculture feed: is cholesterol a hidden treasure for fish nutrition? PLOS ONE $\mathbf{8}$, e81705.

55. Parrish CC (2016) Effect of replacing fish oil and fish meal in aquafeeds with terrestrial products on growth performance and chemical composition in atlantic salmon. In International Symposium of Fish Nutrition and Feeding (ISFNF 2016), Sun Valley, Idaho, USA.

56. Atkinson J, Hilton J \& Slinger S (1984) Evaluation of acidinsoluble ash as an indicator of feed digestibility in rainbow trout (Salmo gairdneri). Can J Fish Aquat Sci 41, 1384-1386.

57. Turchini GM, Francis DS \& De Silva SS (2006) Fatty acid metabolism in the freshwater fish Murray cod (Maccullochella peelii peelii) deduced by the whole-body fatty acid balance method. Comp Biochem Physiol B Biochem Mol Biol 144, 110-118.

58. Turchini G, Francis D \& De Silva S (2007) A whole body, in vivo, fatty acid balance method to quantify PUFA metabolism (desaturation, elongation and beta-oxidation). Lipids $\mathbf{4 2}$, 1065-1071.

59. Rosenlund G, Torstensen BE, Stubhaug I, et al. (2016) Atlantic salmon require long-chain $n-3$ fatty acids for optimal growth throughout the seawater period. J Nutr Sci 5, e19.

60. Føre M, Alver M, Alfredsen JA, et al. (2016) Modelling growth performance and feeding behaviour of Atlantic salmon (Salmo salar L.) in commercial-size aquaculture net pens: model details and validation through full-scale experiments. Aquaculture 464, 268-278.

61. Betancor M, Atalah E, Caballero M, et al. (2011)) $\alpha$-Tocopherol in weaning diets for European sea bass (Dicentrarchus labrax) improves survival and reduces tissue damage caused by excess dietary DHA contents. Aquacult Nutr 17, e112-e122.

62. Glencross B \& Rutherford N (2011) A determination of the quantitative requirements for docosahexaenoic acid for juvenile barramundi (Lates calcarifer). Aquacult Nutr 17, e536-e548.

63. Ostbye TK, Kjaer MA, Rora AMB, et al. (2011) High $n$-3 HUFA levels in the diet of Atlantic salmon affect muscle and mitochondrial membrane lipids and their susceptibility to oxidative stress. Aquacult Nutr 17, 177-190.

64. Ruyter B, Rosjo C, Einen O, et al. (2000) Essential fatty acids in Atlantic salmon: effects of increasing dietary doses of $n-6$ and $n-3$ fatty acids on growth, survival and fatty acid composition of liver, blood and carcass. Aquacult Nutr 6, 119-127.

65. Sales J \& Glencross B (2011) A meta-analysis of the effects of dietary marine oil replacement with vegetable oils on growth, feed conversion and muscle fatty acid composition of fish species. Aquacult Nutr 17, e271-e287.

66. Sanden M, Stubhaug I, Berntssen MH, et al. (2011) Atlantic salmon (Salmo salar L.) as a net producer of long-chain marine $\omega$-3 fatty acids. J Agric Food Chem 59, 12697-12706.

67. Miller MR, Nichols PD \& Carter CG. (2008) n-3 Oil sources for use in aquaculture-alternatives to the unsustainable harvest of wild fish. Nutr Res Rev 21, 85-96.

68. Bell JG, Henderson RJ, Tocher DR, et al. (2004) Replacement of dietary fish oil with increasing levels of linseed oil: modification of flesh fatty acid compositions in Atlantic salmon
(Salmo salar) using a fish oil finishing diet. Lipids 39, 223-232.

69. McKenzie D, Higgs D, Dosanjh B, et al. (1998) Dietary fatty acid composition influences swimming performance in Atlantic salmon (Salmo salar) in seawater. Fish Physiol Biochem 19, 111-122.

70. Sargent JR, Tocher DR \& Bell JG (2003) 4 - The lipids. In Fish Nutrition, 3rd ed., pp. 181-257 [JEHW Hardy, editor]. San Diego: Academic Press.

71. Bell JG, Pratoomyot J, Strachan F, et al. (2010) Growth, flesh adiposity and fatty acid composition of Atlantic salmon (Salmo salar) families with contrasting flesh adiposity: effects of replacement of dietary fish oil with vegetable oils. Aquaculture 306, 225-232.

72. Hixson SM, Parrish CC \& Anderson DM. (2014) Full substitution of fish oil with camelina (Camelina sativa) oil, with partial substitution of fish meal with camelina meal, in diets for farmed Atlantic salmon (Salmo salar) and its effect on tissue lipids and sensory quality. Food Chem 157, 51-61.

73. Stubhaug I, Lie $\varnothing \&$ Torstensen BE. (2007) Fatty acid productive value and $\beta$-oxidation capacity in Atlantic salmon (Salmo salar L.) fed on different lipid sources along the whole growth period. Aquacult Nutr 13, 145-155.

74. Turchini G, Francis D, Senadheera S, et al. (2011) Fish oil replacement with different vegetable oils in Murray cod: evidence of an "omega-3 sparing effect" by other dietary fatty acids. Aquaculture 315, 250-259.

75. Mourente G, Dick JR, Bell JG, et al. (2005) Effect of partial substitution of dietary fish oil by vegetable oils on desaturation and $\beta$-oxidation of [1-14C]18:3n-3 (LNA) and [1-14C]20:5n-3 (EPA) in hepatocytes and enterocytes of European sea bass (Dicentrarchus labrax L.). Aquaculture 248, 173-186.

76. Bell JG, Henderson RJ, Tocher DR, et al. (2002) Substituting fish oil with crude palm oil in the diet of Atlantic salmon (Salmo salar) affects muscle fatty acid composition and hepatic fatty acid metabolism. J Nutr 132, 222-230.

77. Budge SM, Penney SN \& Lall SP (2011) Response of tissue lipids to diet variation in Atlantic salmon (Salmo salar): implications for estimating diets with fatty acid analysis. $J$ Exp Mar Biol Ecol 409, 267-274.

78. Raz A, Kamin-Belsky N, Przedecki F, et al. (1997) Fish oil inhibits $\Delta 6$ desaturase activity in vivo: utility in a dietary paradigm to obtain mice depleted of arachidonic acid. $J$ Nutr Biochem 8, 558-565.

79. Codabaccus BM, Carter CG, Bridle AR, et al. (2012) The " $n-3$ LC-PUFA sparing effect" of modified dietary $n$-3 LC-PUFA content and DHA to EPA ratio in Atlantic salmon smolt. Aquaculture 356-357, 135-140.

80. Eroldoğan TO, Yılmaz AH, Turchini GM, et al. (2013) Fatty acid metabolism in European sea bass (Dicentrarchus labrax): effects of $n$-6 PUFA and MUFA in fish oil replaced diets. Fish Physiol Biochem 39, 941-955.

81. Rombenso AN, Trushenski JT, Jirsa D, et al. (2015) Successful fish oil sparing in white seabass feeds using saturated fatty acid-rich soybean oil and 22: 6n-3 (DHA) supplementation. Aquaculture 448, 176-185.

82. Trushenski J, Mulligan B, Jirsa D, et al. (2013) Sparing fish oil with soybean oil in feeds for White Seabass: effects of inclusion rate and soybean oil composition. North Am J Aqualcult 75, 305-315.

83. Bell JG, McGhee F, Campbell PJ, et al. (2003) Rapeseed oil as an alternative to marine fish oil in diets of post-smolt Atlantic salmon (Salmo salar): changes in flesh fatty acid composition and effectiveness of subsequent fish oil "wash out". Aquaculture 218, 515-528. 
84. Bell JG, Tocher DR, Henderson RJ, et al. (2003) Altered fatty acid compositions in Atlantic salmon (Salmo salar) fed diets containing linseed and rapeseed oils can be partially restored by a subsequent fish oil finishing diet. J Nutr 133, 2793-2801.

85. Sinclair AJ, Attar-Bashi NM \& Li D (2002) What is the role of $\alpha$-linolenic acid for mammals? Lipids 37, 1113-1123.

86. Berge GM, Ruyter B \& Asgard T (2004) Conjugated linoleic acid in diets for juvenile Atlantic salmon (Salmo salar): effects on fish performance, proximate composition, fatty acid and mineral content. Aquaculture 237, 365-380.

87. Codabaccus MB, Bridle AR, Nichols PD, et al. (2011) Effect of feeding Atlantic salmon (Salmo salar L.) a diet enriched with stearidonic acid from parr to smolt on growth and $n$-3 longchain PUFA biosynthesis. Br J Nutr 105, 1772-1782.

88. Bransden MP, Carter CG \& Nichols PD (2003) Replacement of fish oil with sunflower oil in feeds for Atlantic salmon (Salmo salar L.): effect on growth performance, tissue fatty acid composition and disease resistance. Comp Biochem Physiol B Biochem Mol Biol 135, 611-625.

89. Pratoomyot J, Bendiksen EA, Bell JG, et al. (2010) Effects of increasing replacement of dietary fishmeal with plant protein sources on growth performance and body lipid composition of Atlantic salmon (Salmo salar L.). Aquaculture 305, 124-132. 